\title{
Preclinical validation and imaging of Wnt-induced repair in human 3D lung tissue cultures
}

\author{
Franziska E. Uhl ${ }^{1}$, Sarah Vierkotten ${ }^{1}$, Darcy E. Wagner ${ }^{1}$, Gerald Burgstaller ${ }^{1}$, \\ Rita Costa ${ }^{1}$, Ina Koch ${ }^{2}$, Michael Lindner ${ }^{2}$, Silke Meiners ${ }^{1}$, Oliver Eickelberg ${ }^{1}$ and \\ Melanie Königshoff ${ }^{1}$
}

Affiliations: ${ }^{1}$ Comprehensive Pneumology Center, Helmholtz Center Munich, Ludwig Maximilians University Munich, University Hospital Grosshadern, Member of the German Center for Lung Research (DZL), Munich, Germany. ${ }^{2}$ Asklepios Clinics, Gauting, Germany.

Correspondence: M. Königshoff, Comprehensive Pneumology Center, Helmholtz Center Munich, Ludwig Maximilians University Munich, University Hospital Grosshadern, Max-Lebsche-Platz 31, Munich, Germany.

E-mail: melanie.koenigshoffahelmholtz-muenchen.de

ABSTRACT Chronic obstructive pulmonary disease (COPD) is characterised by a progressive loss of lung tissue. Inducing repair processes within the adult diseased lung is of major interest and $\mathrm{Wnt} / \beta$ catenin signalling represents a promising target for lung repair. However, the translation of novel therapeutic targets from model systems into clinical use remains a major challenge.

We generated murine and patient-derived three-dimensional (3D) ex vivo lung tissue cultures (LTCs), which closely mimic the $3 \mathrm{D}$ lung microenvironment in vivo. Using two well-known glycogen synthase kinase-3 $\beta$ inhibitors, lithium chloride $(\mathrm{LiCl})$ and CHIR 99021 (CT), we determined Wnt/ $\beta$-catenin-driven lung repair processes in high spatiotemporal resolution using quantitative PCR, Western blotting, ELISA, (immuno)histological assessment, and four-dimensional confocal live tissue imaging.

Viable 3D-LTCs exhibited preserved lung structure and function for up to 5 days. We demonstrate successful $\mathrm{Wnt} / \beta$-catenin signal activation in murine and patient-derived 3D-LTCs from COPD patients. Wnt/ $\beta$-catenin signalling led to increased alveolar epithelial cell marker expression, decreased matrix metalloproteinase-12 expression, as well as altered macrophage activity and elastin remodelling. Importantly, induction of surfactant protein $\mathrm{C}$ significantly correlated with disease stage (per cent predicted forced expiratory volume in $1 \mathrm{~s}$ ) in patient-derived 3D-LTCs.

Patient-derived 3D-LTCs represent a valuable tool to analyse potential targets and drugs for lung repair. Enhanced Wnt/B-catenin signalling attenuated pathological features of patient-derived COPD 3D-LTCs.

$@$ ERSpublications

Patient-derived 3D-LTCs are a powerful tool for preclinical drug validation and imaging of Wnt-induced lung repair http://ow.ly/L7BCe

This article has supplementary material available from erj.ersjournals.com

Received: Oct 032014 | Accepted after revision: March 222015 | First published online: April 302015

Support statement: This study was supported by a European Research Council Starting Grant to M. Königshoff (ERC-2010-StG 261302) and the Junior Research Group Programme (Helmholtz Association, Berlin, Germany). D.E. Wagner is supported by a Whitaker International Scholar Fellowship and the Helmholtz Munich Postdoctoral Programme. Funding information for this article has been deposited with FundRef.

Conflict of interest: Disclosures can be found alongside the online version of this article at erj.ersjournals.com

Copyright OERS 2015 


\section{Introduction}

Chronic obstructive pulmonary disease (COPD) is a global health problem and will be the third leading cause of death by 2020. In addition to high morbidity with end-stage disease, COPD severely limits the quality of life of affected patients. Most importantly, there is currently no therapy that stabilises or even reverses disease progression [1-3]. COPD leads to progressive distortion of normal lung architecture and impairment of lung function. It is a complex heterogeneous disease with several phenotypes. One major feature of COPD is emphysema, which is defined as destruction of the alveolar surface area for gas exchange due to distal airspace enlargement [4-6]. Chronic inflammation and ongoing proteolysis of the extracellular matrix, as well as structural cell death, have been shown to contribute to disease initiation and progression [6, 7]. Moreover, the inability of the lung to activate self-repair mechanisms in COPD is thought to be a significant cause of the progressive destruction of functional tissue in emphysema. The restoration of functional lung tissue in humans would thus be a tremendous step forward, particularly in light of the fact that the de novo regeneration of the adult lung has not yet been demonstrated $[8,9]$.

The lung epithelium is essential for normal lung function. Besides immune surveillance and particle removal, the epithelium is most important for gas exchange. In the alveoli, the epithelium is mainly composed of alveolar epithelial type (AT)I and II cells. ATII cells are essential for lung growth and serve as progenitors, initiating alveolar epithelial restoration and giving rise to new ATII cells or differentiating into ATI cells $[8,10-12]$. The Wnt/ $\beta$-catenin signalling has a crucial role in lung epithelial development and further represents a promising factor for lung repair $[9,11]$. Embryos lacking Wnt2/2b exhibit complete lung agenesis and conditional inactivation of $\beta$-catenin results in the absence of the lung bud [13]. Furthermore, we and others have demonstrated a crucial role of $\mathrm{Wnt} / \beta$-catenin signalling in alveolar epithelial cell function in chronic lung disease. In COPD, Wnt/ $\beta$-catenin signalling is silenced $[14,15]$. Notably, Wnt/ $\beta$-catenin activation by glycogen synthase kinase (GSK)-3 $\beta$ inhibition prevented, as well as reversed, experimental emphysema in an in vivo animal model [14]. Importantly, whether Wnt/ $\beta$-catenin signal activation represents a valid target to initiate repair in human COPD lung tissue remains unknown.

Translation of novel therapeutic targets from model systems into clinical use still represents a major challenge $[16,17]$. While animal models of lung disease represent excellent and important tools to analyse and validate novel disease mechanisms and potential therapeutic targets in whole living organisms in vivo, their validation in primary human tissue is largely limited to the analysis of single cell types in two-dimensional cultures. We generated and applied viable and functional three-dimensional (3D) ex vivo lung tissue cultures (LTCs) from murine and human lung tissue to investigate and visualise pathological and therapeutic measures of COPD/emphysema in spatio-temporal resolution in a $3 \mathrm{D}$ lung microenvironment. This technique allows the analysis of tissue-level responses to pharmacological perturbation in living human tissue. Moreover, murine 3D-LTCs can be applied to extend mechanistic studies, while reducing overall animal experimentation. We investigated, for the first time, the suitability of $\mathrm{Wnt} / \beta$-catenin activation to initiate repair in patient-derived COPD lung tissue and shed further light onto the mechanism and cellular alterations induced by Wnt/ $\beta$-catenin-driven lung repair in COPD.

\section{Materials and methods}

\section{Human tissue}

The experiments with human tissue were approved by the ethics committee of the Ludwig-Maximillian University (Munich, Germany (project number 455-12)). All samples were provided by the Asklepios Biobank for Lung Diseases (Gauting, Germany (project number 333-10)). Written informed consent was obtained from all subjects. Tumour-free tissue from patients who underwent lung tumour resection was used in these studies. COPD status was diagnosed according to Global Initiative for Chronic Obstructive Lung Disease definition [3]. Lung function measurement was conducted by determining the forced expiratory volume in $1 \mathrm{~s}$ (FEV1) and forced vital capacity using spirometry. The clinical characteristics of all patients in these studies are included in online supplementary table S1. Note that not all patients could be included in all experiments performed in this study. The number of subjects is highlighted in the respective experiments.

\section{Animals}

Pathogen-free female C57Bl/6-N mice (C57BL/6NCrl; Charles River, Sulzfeld, Germany), aged 8-12 weeks, were used in all studies. The Wnt reporter animals, BAT-GAL (B6.Cg-Tg(BAT-lacZ)3Picc/J), TCF/ LEF-green fluorescent protein (GFP) (stock Tg(TCF/Lef1-HIST1H2BB/EGFP)61Hadj/J), and MacGreen (B6N.Cg-Tg(Csf1r-EGFP)1Hume/J) were all from The Jackson Laboratory (Bar Harbour, ME, USA) $[18,19]$. Mice were housed with water and food ad libitum. All experiments were performed in accordance with the guidelines of the ethics committee of the Helmholtz Zentrum Munich (Germany) and approved by the regional council of Upper Bavaria (Germany) (project number 55.2-1-54-2532-168-09). 
Animal model of experimental emphysema

Emphysema was induced as described previously [14]. In short, porcine pancreatic elastase (Sigma, Taufkirchen, Germany) was dissolved in sterile PBS (Gibco, Carlsbad, CA, USA) and applied orotracheally (100 U. $\mathrm{kg}^{-1}$ body weight in $80 \mu \mathrm{L} \mathrm{PBS}$ ). Control mice received $80 \mu \mathrm{L}$ sterile PBS. The 3D-LTCs were generated 7 days after the instillation, as described below.

\section{Generation of human and murine 3D-LTCS}

A schematic overview of the process is given in online supplementary figure S1. The procedure was performed under sterile conditions. For the murine 3D-LTCs, healthy and emphysematous mice were anaesthetised with a mixture of ketamine (Bela-Pharm, Vechta, Germany) and xylazine hydrochloride (CP-Pharma, Burgdorf, Germany). After intubation and dissection of the diaphragm, lungs were flushed via the heart with sterile sodium chloride solution and a sample of bronchoalveolar lavage fluid (BALF) was taken $(2 \times 500 \mu \mathrm{L}$ sterile PBS) for all animals that had been instilled with PBS or elastase. In the experiments with nontreated animals, no BALF was taken. Using a syringe pump, lungs were infiltrated with warm, low gelling temperature agarose ( $2 \%$ by weight, A9414; Sigma; kept at $\left.40^{\circ} \mathrm{C}\right)$ in sterile cultivation medium (DMEM/Ham's F12; Gibco, supplemented with $100 \mathrm{U} \cdot \mathrm{mL}^{-1}$ penicillin, $100 \mu \mathrm{g} \cdot \mathrm{mL}^{-1}$ streptomycin and $2.5 \mu \mathrm{g} \cdot \mathrm{mL}^{-1}$ amphotericin B; Sigma). The trachea was ligated with thread to retain the agarose inside the lung. The lung was excised, transferred into a tube with cultivation medium and cooled on ice for 10 min to allow gelling of the agarose. The lobes were separated and cut with a vibratome (Hyrax V55; Zeiss, Jena, Germany) to a thickness of $300 \mu \mathrm{m}$ using a speed of $10-12 \mu \mathrm{m} \cdot \mathrm{s}^{-1}$, a frequency of $80 \mathrm{~Hz}$ and an amplitude of $1 \mathrm{~mm}$. Agarose was largely washed out upon slicing. The 3D-LTCs were cultivated in medium supplemented with $0.1 \%$ fetal calf serum (FCS) (PAA, Fairfield, CT, USA). The slices floated in the media over the culture period. Individual 3D-LTCs were cultivated at $37^{\circ} \mathrm{C}$ in humidified conditions containing 5\% (volume/ volume) $\mathrm{CO}_{2}$ in 24-well plates under submerged conditions with changes of medium every other day.

For the patient-derived 3D-LTCs, lung segments from non-COPD and COPD patients without tumour infiltration were cannulated via a visible bronchus and filled with warm agarose (3wt-\%) immediately after excision. Lung segments were cooled on ice for $30 \mathrm{~min}$ to allow gelling of the agarose, cut to a thickness of $500 \mu \mathrm{m}$ using a speed of $6-10 \mu \mathrm{m} \cdot \mathrm{s}^{-1}$, a frequency of $100 \mathrm{~Hz}$ and an amplitude of $1.2 \mathrm{~mm}$. Biological exclusion criteria included lobes with known cancer; and logistically we did not accept any lobes that did not have intact bronchioles, as we could not fill these lobes with agarose for subsequent cutting.

For live/dead staining, WST-1 assay and ELISA, punches of 3D-LTCs were generated for standardisation and quantitative assessment. 3D-LTCs were punched to a diameter of $4 \mathrm{~mm}$ using a biopsy punch, ensuring exclusion of major airways. Punches were taken from similar peripheral areas of the lung to minimise sample variation and standardise for tissue volume in further quantitative analysis.

\section{Cell culture}

MH-S murine alveolar macrophages (ATCC CRL-2019) and MLE12 murine lung epithelial cells (ATCC CRL-2110) were maintained in RPMI 1640 (Life Technologies, Carlsbad, CA, USA) supplemented with $10 \%$ FCS, $100 \mathrm{U} \cdot \mathrm{mL}^{-1}$ penicillin and $100 \mu \mathrm{g} \cdot \mathrm{mL}^{-1}$ streptomycin. Cells were seeded at $\sim 80 \%$ confluency ( 300000 cells per well) in a 24 -well plate and allowed to adhere for $24 \mathrm{~h}$. Cells were then serum starved $(0.1 \%$ FCS $)$ for $24 \mathrm{~h}$ to allow for cell synchronisation prior to treatment. Cells were then treated for $24 \mathrm{~h}$ with $0,2,10$ or $20 \mathrm{mM}$ lithium chloride ( $\mathrm{LiCl}$ ) in $0.1 \%$ FCS-supplemented RPMI medium.

\section{Wnt/ $\beta$-catenin activation by LiCl and CHIR 99021}

Wnt/ $\beta$-catenin activation was induced in murine and human 3D-LTC using two well-known GSK-3 $\beta$

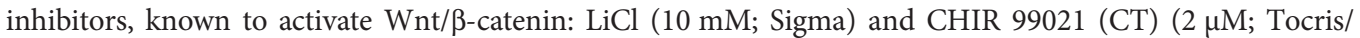
R\&D, Minneapolis, MN, USA). Inhibition of GSK-3 $\beta$ activity causes intracellular accumulation of $\beta$-catenin, a feature associated with the canonical Wnt/ $\beta$-catenin signalling pathway $[20,21]$. Consecutive slices/punches from murine and human 3D-LTCs were used for treatments and controls.

\section{Live/dead staining}

Punched 3D-LTC (more than two punches per animal/patient and treatment) were incubated with cultivation medium supplemented with $2 \mu \mathrm{M}$ calcein-AM and $4 \mu \mathrm{M}$ ethidium homodimer-1 (Life Technologies). After $40 \mathrm{~min}$, samples were washed, fixed with $4 \%$ paraformaldehyde and imaged with a confocal microscope (LSM 710; Zeiss).

WST-1 assay

Each 3D-LTC punch was incubated with $200 \mu \mathrm{L}$ cultivation medium supplemented with WST-1 $15 \mu \mathrm{L} \cdot \mathrm{mL}^{-1}$ (Roche, Mannheim, Germany) for mouse $3 \mathrm{D}$-LTCs or $30 \mu \mathrm{L} \cdot \mathrm{mL}^{-1}$ for patient-derived 3D-LTCs. After $2 \mathrm{~h}, 150 \mu \mathrm{L}$ supernatant was removed and measured in a plate reader at $450 \mathrm{~nm}$. Reference 
measurements at $690 \mathrm{~nm}$ were subtracted to correct for nonspecific absorbance. For each time point and treatment, punched 3D-LTCs from at least three different mice, or different tissue areas of individual patient-derived 3D-LTCs per patient, were analysed. All samples were normalised to the mean value of day 0 treatments $(100 \%)$.

Following treatment with $0,2,10$ or $20 \mathrm{mM} \mathrm{LiCl}$, MHS and MLE12 cells were incubated for $1 \mathrm{~h}$ with WST-1 $100 \mu \mathrm{L} \cdot \mathrm{mL}^{-1}$ cultivation media. $100 \mu \mathrm{L}$ supernatant was transferred and absorbance was measured as detailed earlier.

\section{RNA isolation}

3D-LTCs were washed twice with PBS, snap frozen in liquid nitrogen and pulverised using a microdismembrator S (Thermo Fisher Scientific, Darmstadt, Germany). Afterwards, the Perfect Pure RNA Fibrous Tissue Kit (5 Prime, Hilden, Germany) was used with modifications of the protocol provided by the manufacturer. Three to five slices per sample from different lung regions or lobes were used to increase the representativeness of the tissue. Each RNA isolation contained slices from one to two animals and each experiment was performed using samples from at least two separate elastase instillations. RNA concentration was determined using a Nano-Drop spectrophotometer (Thermo Fisher Scientific). For human samples, RNA quality was assessed by capillary electrophoresis using a Bioanalyzer 2100 (Agilent Technology, Oberhaching, Germany).

\section{Relative quantitative PCR}

Relative quantitative (q)PCR was performed using SYBR Green (Roche) and a LC480 Light Cycler (Roche), as described previously [14]. HPRT was used as the reference gene. PCR was performed using the primers listed in online supplementary tables S2 and S3 at a final concentration of $500 \mathrm{nM}$, with annealing temperatures between 56 and $60^{\circ} \mathrm{C} . \Delta \mathrm{Cp}$ is defined as the relative transcript abundance of a gene $(\Delta \mathrm{Cp}=\mathrm{Cp}$ HPRT $-\mathrm{Cp}$ target gene).

\section{Western blotting}

Samples were processed and homogenised as described in the section on RNA isolation. Proteins were extracted using a tissue protein extraction reagent buffer (Thermo Fisher Scientific) supplemented with proteinase and phosphatase inhibitors (Roche). Protein content of 3D-LTCs was measured using the Pierce BCA Protein Assay Kit (Thermo Fisher Scientific). For blotting, nitrocellulose membranes were used. After blocking with 5 wt- $\%$ nonfat dry milk (AppliChem) for $1 \mathrm{~h}$, blots were incubated with primary antibodies at $4^{\circ} \mathrm{C}$ overnight or for $1.5 \mathrm{~h}$ at room temperature. Secondary antibodies were incubated for $1.5 \mathrm{~h}$. For detection, an enhanced chemoluminescence substrate (Thermo Fisher Scientific) was used and blots were imaged using a ChemiDoc XRS+ (BioRad, Hercules, CA, USA). The antibodies used are listed in online supplementary table S4.

\section{Surfactant protein C ELISA}

To quantify secreted surfactant protein C (SFTPC) content, an ELISA system (Cusabio, Wuhan, China) was used according to the protocol provided by the manufacturer. SFTPC content was displayed as ratio to total protein determined using the Pierce BCA Protein Assay Kit.

\section{Immunofluorescence}

3D-LTCs were fixed with acetone/methanol (AppliChem, Darmstadt, Germany) 50:50 by volume for $20 \mathrm{~min}$, punched to a diameter of $4 \mathrm{~mm}$, using the same criteria described in the section on live/dead staining, blocked for $1 \mathrm{~h}$ with $5 \%$ by weight bovine serum albumin (Sigma) in PBS, and incubated with primary antibody overnight at $4^{\circ} \mathrm{C}$. After incubation with a secondary antibody for $1-2 \mathrm{~h}$, staining was evaluated via confocal microscopy. 3D reconstruction was conducted using the IMARIS $\times 64$ software (version 7.6.4; Bitplane, Zurich, Switzerland). Antibodies used are listed in online supplementary table S5.

For semi-quantitative analysis of apoptosis in the 3D-LTCs at different time points, $4 \mathrm{~mm}$ punches ( $\mathrm{n}=3$ for each time point) were prepared and stained with cleaved caspase 3 antibody and 4'-6-diamidino-2-phenylindole (DAPI). Confocal $\mathrm{z}$-stack images were taken at $100 \times$ magnification using a tile scan $(2 \times 2)$ to obtain the maximum area of each punch. A maximum intensity image was generated with the Zen software and the cleaved caspase 3 positive stained cells were quantified relative to the total amount of cells determined via DAPI using the IMARIS $\times 64$ software.

\section{Histology and immunohistochemistry}

Immunohistochemical stainings were performed as described previously [14]. In short, 3D-LTCs were fixed with $4 \%$ (weight/volume) paraformaldehyde overnight, and paraffin embedded. 3- $\mu \mathrm{m}$ sections were cut using a microtome (Zeiss), mounted on glass slides and subjected to antigen retrieval. After deparaffinisation and rehydration, staining was performed according to standard protocols for haematoxylin and eosin (H\&E), 
Masson's trichrome and Hart's stain (elastin). Finally, samples were mounted using HI-MO mounting medium (Bio-Optica, Milan, Italy) and covered with a cover slip. Microscopic scanning of the slides was conducted with a Mirax scanner (Zeiss) and representative images are displayed in all figures.

\section{LacZ staining}

LacZ staining was performed as previously described [22]. In short, 3D-LTCs derived from BAT-GAL mice were washed twice with PBS and prefixed for $10 \mathrm{~min}$ in $4 \%$ paraformaldehyde. After washing, the 3D-LTCs were incubated in staining solution $(5 \mathrm{mM}$ potassium ferricyanide, $5 \mathrm{mM}$ potassium ferrocyanide, $2 \mathrm{mM}$ magnesium chloride hexahydrate, $65 \mathrm{mM}$ sodium phosphate dibasic and $15 \mathrm{mM}$ sodium phosphate monobasic (all Sigma) and $1 \mathrm{mg} \cdot \mathrm{mL}^{-1}$ 5-bromo-4-chloro-3-indolyl- $\beta$-D-galactopyranoside (X-Gal; Research Products International, Mount Prospect, IL, USA) in the dark at $37^{\circ} \mathrm{C}$ overnight. X-gal staining results in turquoise staining in cells in which canonical Wnt signalling has been activated. On the following day, 3D-LTCs were washed and mounted as described for immunohistochemical staining.

\section{Confocal live cell imaging}

Confocal time-lapse microscopy was conducted on an LSM710 system (Zeiss) containing an inverted AxioObserver.Z1 stand. 3D-LTCs were maintained submerged in DMEM/Ham's F12 medium, containing $0.1 \%$ FCS and $15 \mathrm{mM}$ HEPES (Gibco), over the period of observation. Imaging of the 3D-LTCs was performed using imaging plates (BD, Heidelberg, Germany) in a PM S1 incubator chamber (Zeiss) at $37^{\circ} \mathrm{C}$. 3D-LTCs were immobilised using an autoclaved stainless steel ring. Microscopy was conducted in the middle of the slice. Image acquisition began within $30 \mathrm{~min}$ of the transfer of the 3D-LTCs to the imaging plates. Z-stacks $(50-100 \mu \mathrm{m})$ from the middle of the 3D-LTCs were acquired using an EC Plan-Neofluar DICII $20 \times / 0.8$ na objective lens (Zeiss) at 30-min intervals up to $24 \mathrm{~h}$. The automated time-lapse microscope was operated by ZEN2009 software (Zeiss). The confocal four-dimensional data sets were imported into Imaris 7.4.0 software (Bitplane) and processed by applying a volume-rendering algorithm.

Imaging of the time-lapse videos of the MacGreen animals was performed using $35 \mathrm{~mm}$ CellView cell culture dishes (Greiner BioOne, Frickenhausen, Germany). The 3D-LTCs were spatially fixed by tissue culture inserts (ThinCerts, 8- $\mu \mathrm{m}$ pore size; Greiner Bio-One) and the lid of the CellView cell culture dish was tightly sealed with parafilm. 3D-LTCs were then imaged with or without $10 \mathrm{mM} \mathrm{LiCl}$ treatment. A single z-plane from the middle of each tissue slice was selected from the bright field acquisition for spatial orientation of the duration of the experiment using Zen software. A maximum projection intensity of the green channel was generated and overlaid on the single z-plane from the bright field image. Cell track length and speed was analysed using the Track Spots algorithm in IMARIS with an estimated spot detection diameter of 5.5-20 $\mu \mathrm{m}$.

\section{Statistical analysis}

All data are presented as mean \pm SD and calculated using GraphPad Prism 5 (GraphPad Software, La Jolla, CA, USA). For comparison of two groups, a paired t-test and, if not applicable an unpaired t-test with Welch's correction was used. For calculation of significances of $\log$ fold changes, a one-sample t-test comparing to a hypothetical value of 0 was used. Comparisons of more than two groups were performed using ANOVA and the Bonferroni post hoc test. For the correlation analysis, the nonparametric method (Spearman) was used due to the assumed non-Gaussian distribution and small sample size of the human samples. $\mathrm{p}<0.05$ was considered statistically significant.

\section{Results}

\section{Viability and functionality of murine 3D ex vivo lung tissue cultures}

The 3D-LTCs were generated from both normal and diseased murine tissue, as well as patient-derived lung tissue. A schematic overview of the process is depicted in online supplementary figure S1. 3D-LTCs from healthy and emphysematous animals ( $300 \mu \mathrm{m}$ thickness, fig. 1a) remained viable over a time period of 5 days as determined by live/dead staining (fig. 1b) and exhibited a slight initial increase in metabolic activity as determined by WST-1 measurement (online supplementary fig. 2a). Importantly, cellular function was also maintained as analysed by live cell imaging of bronchial epithelial cells, showing sustained ciliary beating at day 7 (online supplementary video S1). As we are particularly interested in alveolar epithelial cell function, we further determined SFTPC secretion as a measure of ATII cell function, which was comparable over the period of 5 days, but exhibited a significant drop at day 7 and day 14 in culture (fig. 1c). In order to assess whether alveolar epithelial cell death is responsible for this decline in SFTPC secretion, we performed cleaved caspase staining over the culture time and found a general increase of apoptotic cells, in particular at day 7 compared to day 5. However, several cell populations were affected (fig. 1d, arrows indicate structural cells within the bronchial (yellow) and alveolar (red) area, as well as apoptotic non-structural cells, potentially leukocytes (white), and online supplementary fig. 2b). This was further confirmed by an increasing number of fragmented nuclei at day 7, as determined by H\&E staining in 3D-LTCs (online supplementary fig. 2c). In parallel with cell 
a)

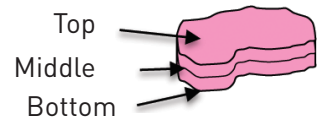

Healthy

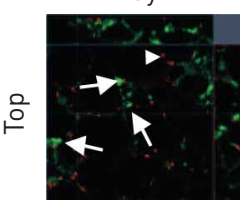

Day 5
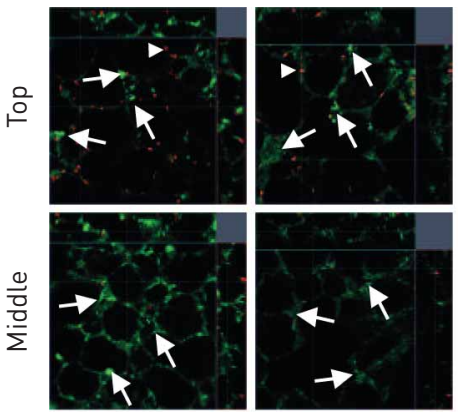

$\varepsilon$
$\stackrel{0}{+}$
$\stackrel{+}{\circ}$
$\infty$
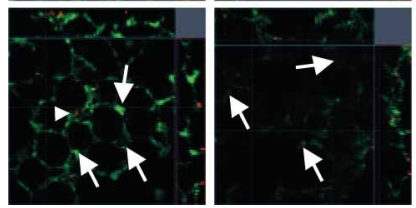

Day 5

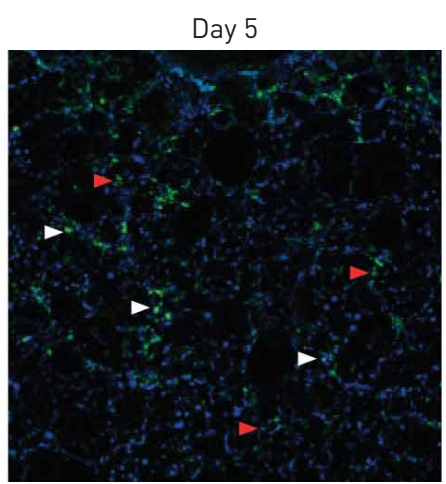

Day 3

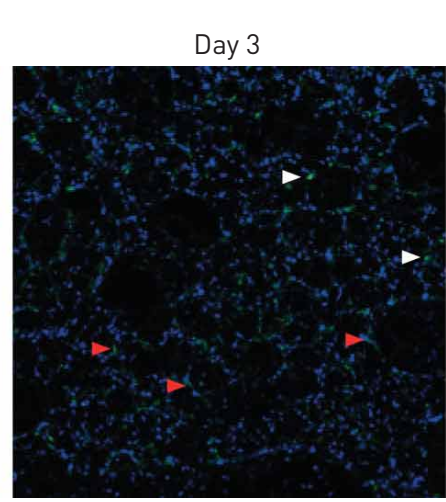

Day 1

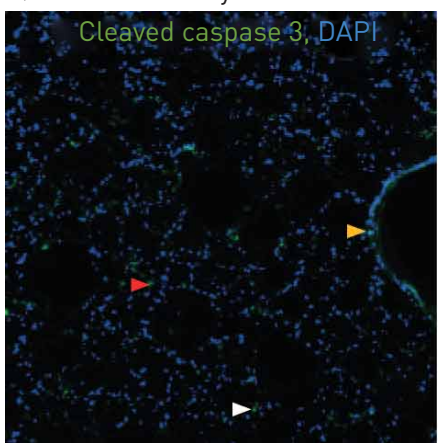

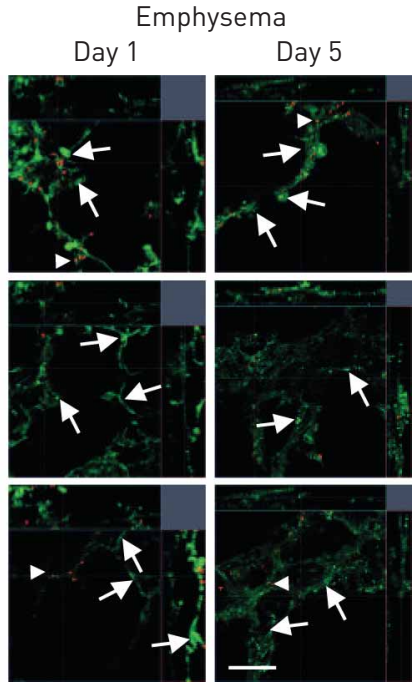

Day 7

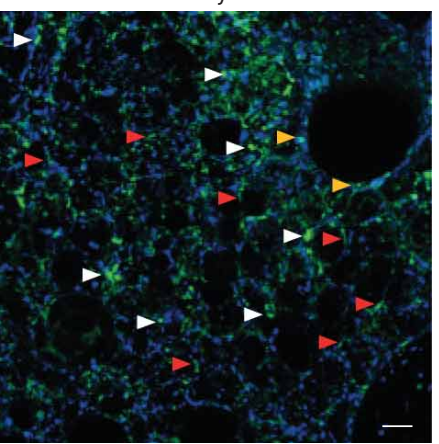

FIGURE 1 Murine three-dimensional (3D) ex vivo tissue cultures (LTC) are viable over culture time. a) Scheme of the different regions of the 3D-LTCs referred to as top, middle and bottom planes. b) Representative live/dead confocal images of the different planes of 3D-LTCs from healthy and emphysematous C57BI/6 mice at the indicated time points. Ortho views of the top, middle and bottom planes. Healthy cells are indicated by green cytoplasm (arrows) and dead cells by red nuclei (arrowheads). c) Quantification of surfactant protein C (SFTPC) secretion of 3D-LTCs from healthy C57Bl/6 mice at different time points of cultivation. $n=8 .{ }^{*}: p<0.05$ compared to previous time point; ${ }^{*}$ : $p<0.05$ compared to day 1. d) Cleaved caspase 3 staining of 3D-LTCs from healthy C57Bl/6 mice during cultivation. Cleaved caspase 3 positive cells are indicated by arrowheads. Arrows indicate structural cells within the bronchial (yellow) and alveolar (red) areas, as well as apoptotic nonstructural cells, potentially leukocytes (white). Nuclei are stained blue (4'-6-diamidino-2-phenylindole (DAPI)). Scale bars=50 $\mu \mathrm{m}$.

viability and function, we characterised the lung structure over the culture period using histology and 3D confocal tissue imaging. Importantly, healthy as well as pathophysiologic lung architecture of diseased murine lung (emphysematous) tissue was preserved over the culture time, as analysed by immunofluorescence (collagen I, CD45, pro-SFTPC, aquaporin 5, podoplanin and E-cadherin) and histological staining (H\&E, Masson's trichrome and Hart's staining; fig. 2a-c and online supplementary fig. 3). Based on these findings, we performed all further experiments over a maximum cultivation period of 5 days.

Therapeutic activation of Wnt/ $\beta$-catenin signalling in murine emphysematous 3D-LTCS

Aberrant Wnt/ $\beta$-catenin signalling has previously been implicated in the development and progression of chronic lung disease $[14,22-24]$. Wnt/ $\beta$-catenin signalling has been found to be downregulated in human small airway cells from COPD patients [15], as well as in a murine model of emphysema [14]. We first aimed to investigate whether these disease-related pathway alterations are maintained and can be modified in ex vivo conditions. 3D-LTCs were generated from emphysematous mice 7 days post-elastase treatment, which is characterised by reduced inflammatory processes and established airspace enlargement (online supplementary fig. S4) [14]. We analysed the transcript level of several Wnt target genes and Wnt pathway components and observed a significant decrease in expression of the Wnt target genes Axin2 and Nkd1, the Wnt receptor Fgfr2, as well as the Wnt ligand Wnt2, while the Wnt signal inhibitor Dkk2 was increased in 3D-LTCs from 
a)
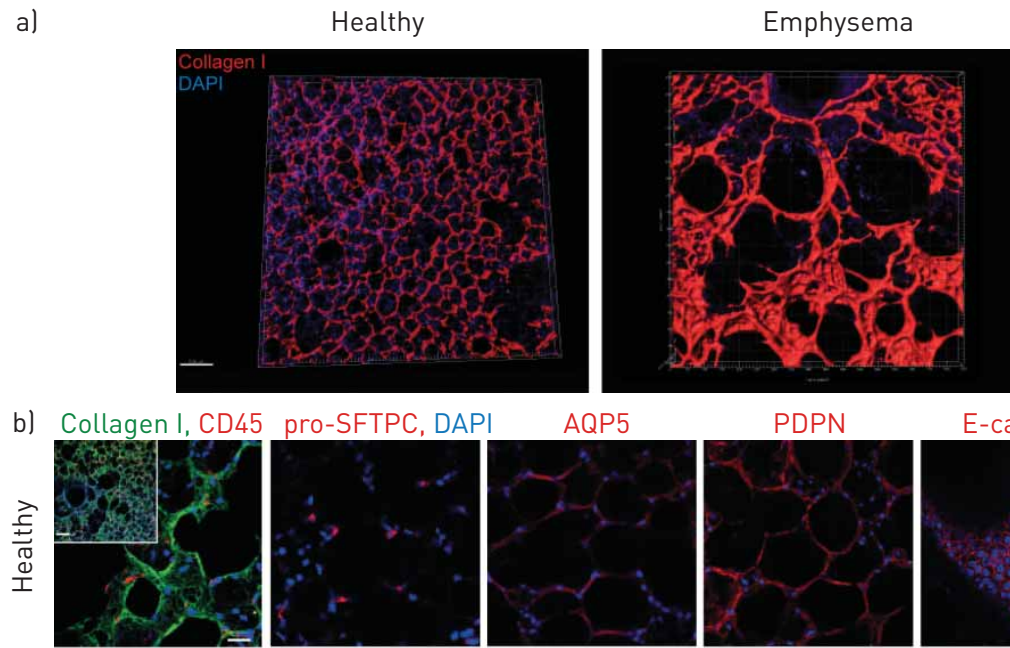

PDPN

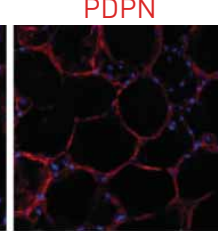

E-cadherin
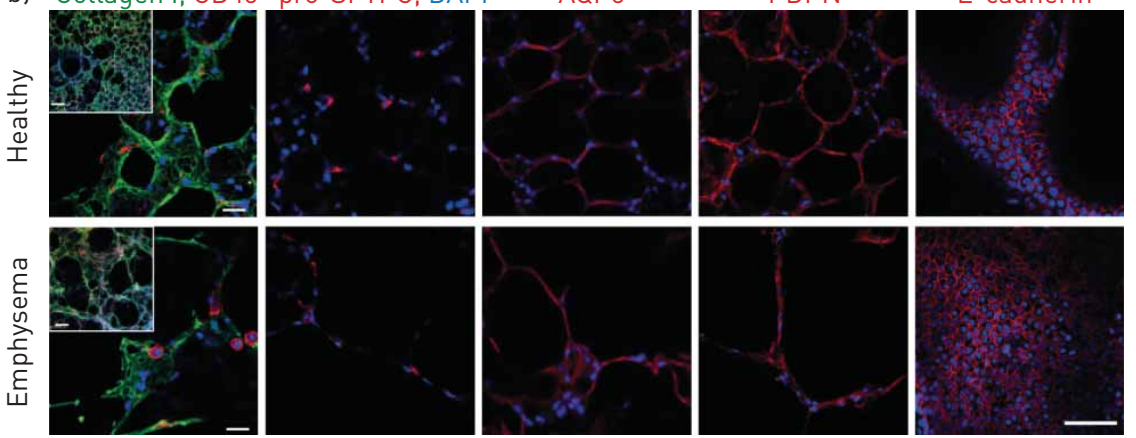

c) Haematoxylin and eosin
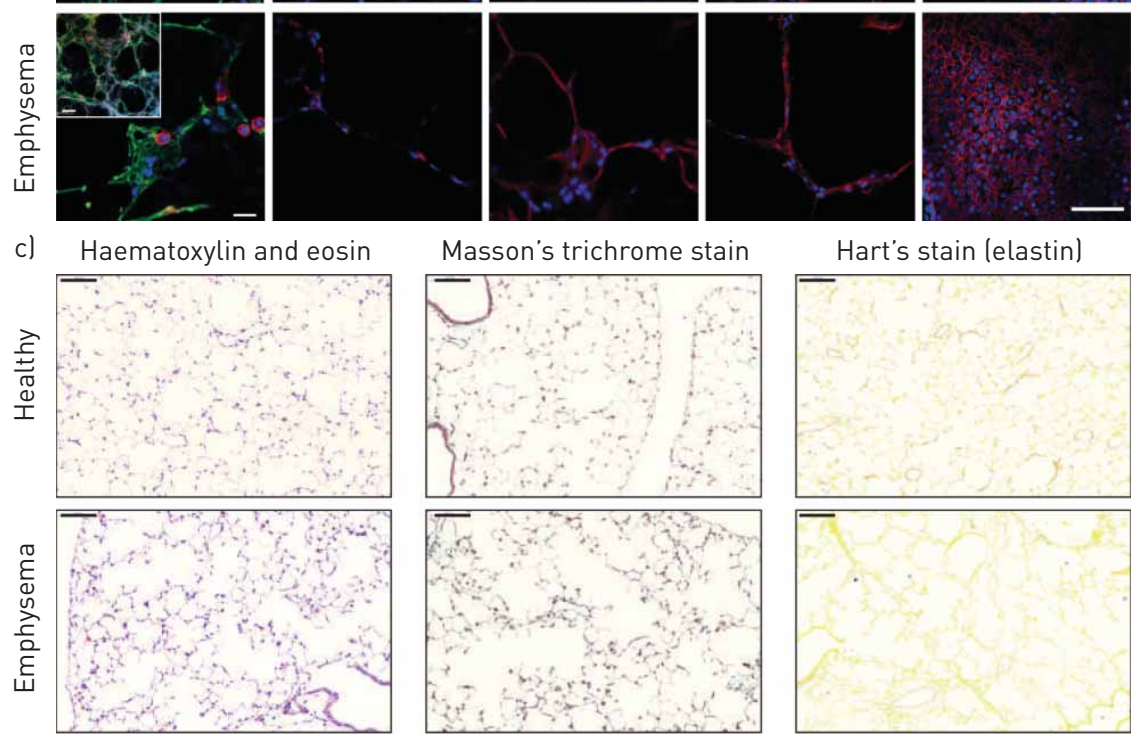

\section{Hart's stain (elastin)}

Axin2

Nkd1
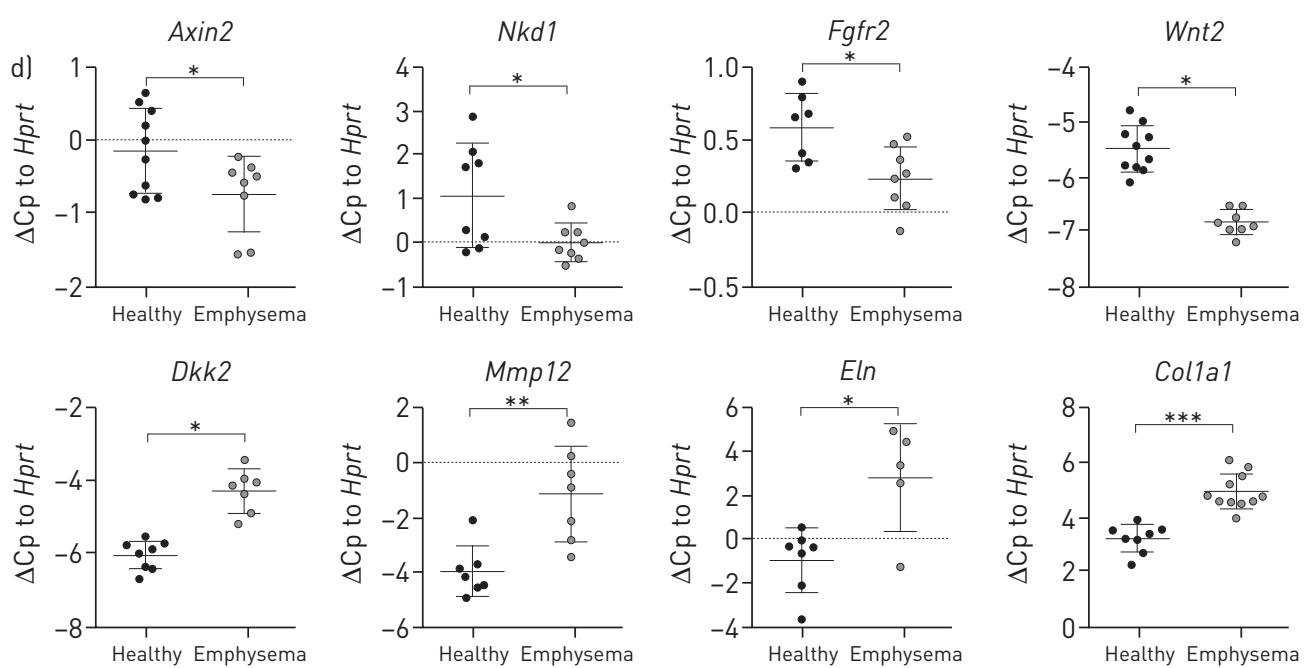

FIGURE 2 Altered lung structure and Wnt signal reduction is preserved in emphysematous three-dimensional (3D) ex vivo tissue cultures (LTCs). a) 3D reconstruction of immunofluorescence-labelled 3D-LTCs from healthy and emphysematous C57BV/6 animals. Collagen I staining; nuclei stained with 4'-6-diamidino-2-phenylindole (DAPI). Scale bar=100 $\mu \mathrm{m}$. b) Immunofluorescence staining of 3D-LTCs from healthy and emphysematous C57Bl/6 mice cultivated for $24 \mathrm{~h}$, showning collagen type I, CD45, pro-surfactant protein C (SFTPC), aquaporin (AQP)5, podoplanin (PDPN) and E-cadherin staining. Nuclei are stained blue with DAPI. Scale bars $=50 \mu \mathrm{m}$. c) Haematoxylin and eosin (H\&E), Masson's trichrome and elastin staining of 3D-LTCs from healthy and emphysematous C57BI/6 mice directly after slice generation. Scale bars $=100 \mu \mathrm{m}$. $\mathrm{d}$ ) Gene expression analysis by quantitative PCR of Wnt target genes Axin2, Nkd1 and Fgfr2, Wnt ligand Wnt2, Wnt antagonists Dkk2 and Mmp12 and the extracellular matrix components Eln and Col1a1 in 3D-LTCs from control and emphysematous C57Bl/6 mice immediately after slicing. Hprt was used as the reference gene. $\mathrm{n}=7-10$. Data are presented as mean \pm SD.*: $p<0.05 ;{ }^{* *}: p<0.01 ;{ }^{* * *}: p<0.001$. 

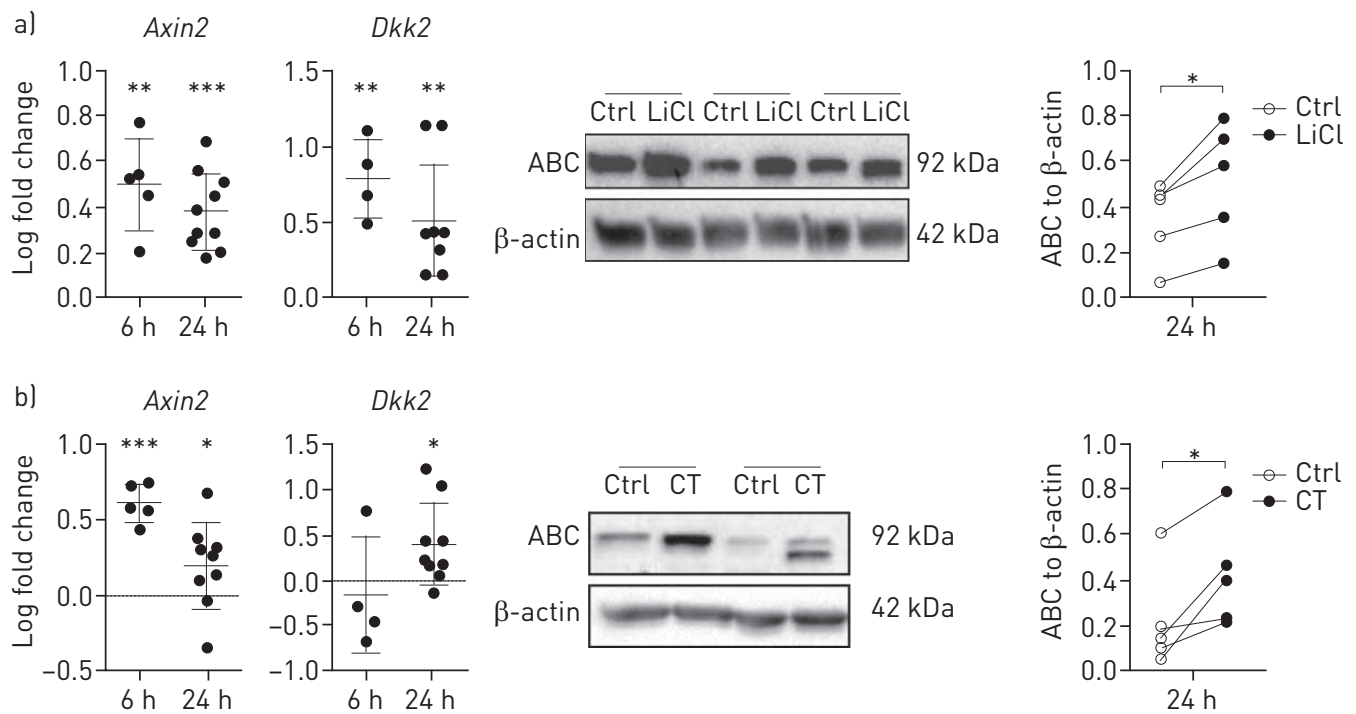

c)
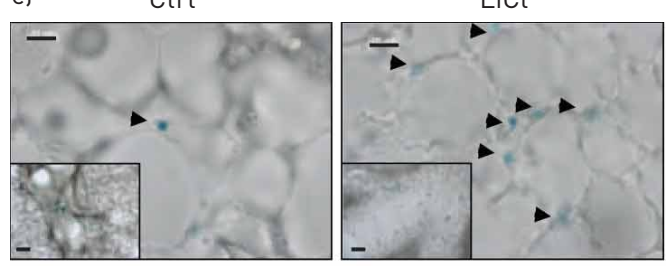

d)

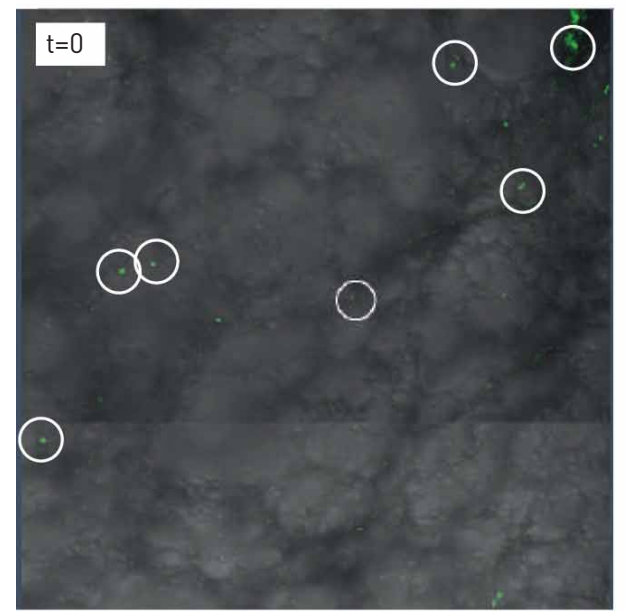

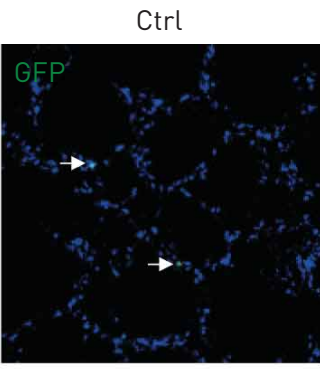
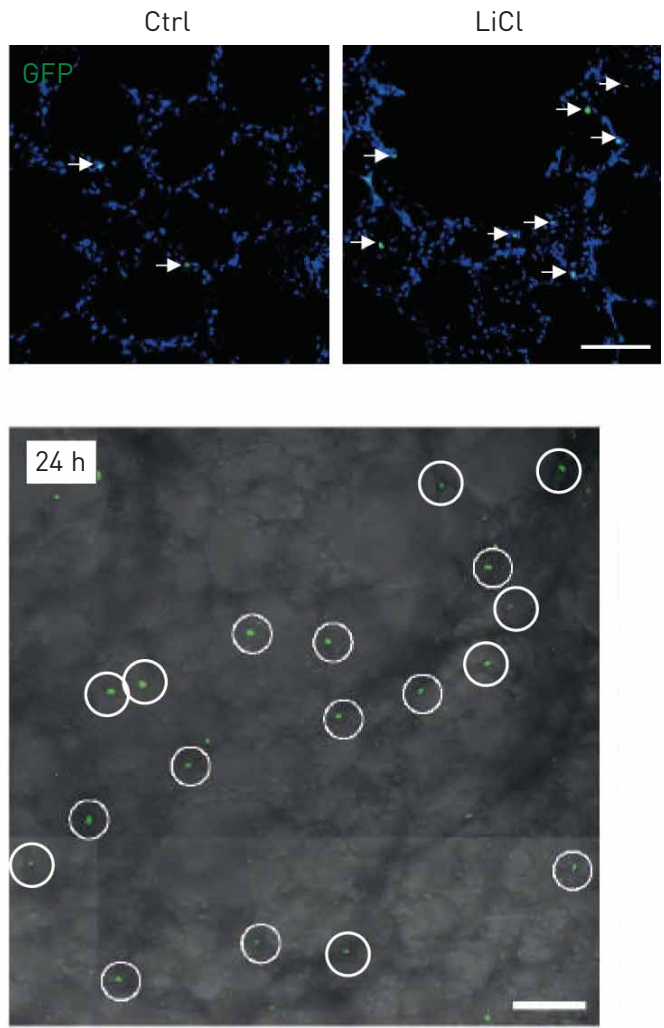

FIGURE 3 Wnt/B-catenin signalling is activated by lithium chloride (LiCl) and CHIR 99021 (CT) in three-dimensional (3D) ex vivo tissue cultures (LTCs) from emphysematous mouse lungs. Gene expression analysis of Wnt target genes Axin2 and Dkk2 by quantitative PCR, and representative Western blot and densitometry of active $\beta$-catenin $(A B C)$ protein expression in 3D-LTCs from emphysematous C57BV/6 mice at indicated time points after treatment with a) $10 \mathrm{mM} \mathrm{LiCl}$ or b) $2 \mu \mathrm{M}$ CT. Dimethylsulfoxide (2 $\mu \mathrm{m}$; Sigma, Taufkirchen, Germany) was used as a control for CT treatment. $n=4-10 .^{*}: p<0.05 ;{ }^{* *}: p<0.01 ;{ }^{* * *}: p<0.001$. c) Left: representative LacZ staining of 3D-LTCs from emphysematous BAT-GAL Wnt-reporter animals after $3 \mathrm{~h}$ of treatment with $0.1 \%$ fetal calf serum (FCS) control medium (Ctrl) or $10 \mathrm{mM} \mathrm{LiCl}$. Activated cells are stained in blue (arrowheads); scale bar $=20 \mu \mathrm{m}$. Inset: activated cells in bronchial regions; scale bar $=100 \mu \mathrm{m}$. Right: representative confocal images of 3D-LTCs from emphysematous TCF/green fluorescent protein (GFP) Wnt-reporter animals after $72 \mathrm{~h}$ of treatment with $0.1 \% \mathrm{FCS}$ control medium or $10 \mathrm{mM}$ LiCl. Activated cells are expressing GFP (arrows). Scale bar=50 $\mu \mathrm{m}$. d) Four-dimensional confocal live cell imaging of 3D-LTCs from emphysematous TCF/GFP Wnt-reporter animals at start of imaging and after $24 \mathrm{~h}$ treatment with $10 \mathrm{mM} \mathrm{LiCl}$. Wnt/ $\beta$-catenin activated cells (expressing GFP) are shown within white circles. Scale bar $=100 \mu \mathrm{m}$. See also online supplementary video S2.

emphysematous mice (fig. 2d). Moreover, well-known pathological measures of human emphysema and murine emphysema models were preserved, including an upregulation of matrix metalloproteinase 12 (Mmp12), elastin (Eln) and collagen I (Col1a1) transcripts in 3D-LTCs from emphysematous mice (fig. 2d) [25, 26]. 
Next, we asked whether Wnt/B-catenin signalling can be re-activated in 3D-LTCs. To this end, we applied murine 3D-LTCs to investigate the feasibility of two different compounds to induce $\mathrm{Wnt} / \beta$-catenin signal activation, $\mathrm{LiCl}(10 \mathrm{mM})$ and $\mathrm{CT}(2 \mu \mathrm{M})$ [27]. Importantly, we did not observe toxicity upon application of $10 \mathrm{mM} \mathrm{LiCl}$ or $2 \mu \mathrm{M} \mathrm{CT}$ in 3D-LTCs from healthy and emphysematous mice ex vivo, which was comparable to two-dimensional cultures of epithelial cell lines, while the MHS macrophage cell line demonstrated increased cellular toxicity, as reported previously (online supplementary fig. 5 and [28]). $\mathrm{LiCl}$ and CT treatment led to increased expression of $\mathrm{Wnt} / \beta$-catenin target genes, such as Axin2 or Dkk2, as quantified by $\mathrm{qPCR}$, as well as significantly increased expression of active $\beta$-catenin protein expression (fig. $3 \mathrm{a}$ and b), as a surrogate marker for active canonical Wnt signalling. These findings are in accordance with previous in vivo studies in a mouse model of experimental emphysema [14], thus highlighting the 3D-LTCs as a suitable tool to model and further expand on lung disease-related mechanism and function in vivo.

\section{Initiation of alveolar epithelial cell repair and reduction of emphysema-related mechanisms by} therapeutic Wnt/B-catenin activation in murine 3D-LTCs

Next, we used 3D-LTCs from two different Wnt reporter mice (BAT-GAL and TCF/LEF-GFP) $[18,19]$ to further elucidate the cellular effect and mechanism upon Wnt/ $\beta$-catenin activation. Wnt $/ \beta$-catenin activated cells were mainly identified within the bronchial epithelium as well as in the alveolar parenchyma, as analysed by LacZ staining (fig. $3 c$ left) as well as GFP expression (fig. 3c right). Notably, live 3D-LTC imaging allowed spatiotemporal identification of Wnt/ $\beta$-catenin-activated lung cells in response to $\mathrm{LiCl}$ treatment (fig. 3d and online supplementary video S2). Wnt/ $\beta$-catenin-activated cells in the lung parenchyma largely stained positive for the ATI cell marker podoplanin (PDPN: T1- $\alpha$ ) and the ATII cell marker pro-SFTPC (fig. 4a), but we did not observe a large number of Wnt/ $\beta$-catenin-activated mesenchymal or endothelial cells (data not shown). We observed a significantly increased expression of PDPN mRNA and protein expression upon Wnt/ $\beta$-catenin activation by $\mathrm{LiCl}$ in murine 3D-LTCs (fig. $4 \mathrm{~b}$ and $\mathrm{d}$ ). Moreover, we detected a decrease in homeodomain-only protein (HOP)X mRNA, a recently described alveolar epithelial cell marker, which might repress surfactant production (fig. 4d) [29, 30]. However, we did not observe significant changes in SFTPC mRNA or protein (fig. $4 \mathrm{c}$ and d). We further elucidated the effect of $\mathrm{Wnt} / \beta$-catenin activation by $\mathrm{LiCl}$ on pathological changes of $\mathrm{Mmp} 12$ and elastin in elastase-induced emphysema. Importantly, both $M m p 12$ and Eln transcripts were significantly decreased in emphysematous 3D-LTCs upon $\mathrm{LiCl}$ or CT treatment, respectively (fig. $4 \mathrm{~d}$ and online supplementary fig. S6). Our data indicate that epithelial cells are the prime responder cells to Wnt/ $\beta$-catenin activation (figs $3 \mathrm{~d}$ and $4 \mathrm{a}$ ), suggesting that the observed effect is primarily on epithelial cell-derived Mmp12 and Eln expression; however, direct or indirect effects on other cell types such as macrophages cannot be excluded. On this line, while we did not observe any macrophage toxicity or apoptosis upon Wnt/ $\beta$-catenin activation by $\mathrm{LiCl}$ in 3D-LTCs (online supplementary fig. S4), we monitored macrophage movement by live cell imaging using MacGreen animals. Interestingly, we found a significant decrease in macrophage movement (as analysed by track length and speed) in LiCl-treated 3D-LTCs from healthy and emphysematous animals, compared to control treatment (online supplementary fig. S7 and videos S3 and S4).

\section{Preclinical validation of therapeutic Wnt/B-catenin signal activation in patient-derived 3D-LTCS}

Based on the findings that murine 3D-LTCs closely reflect aspects of the in vivo situation and represent a powerful tool to further determine the mechanism of $\mathrm{Wnt} / \beta$-catenin activation in living lung tissue, we aimed to translate our findings to patient-derived 3D-LTCs, the use of which is the primary goal for future preclinical drug validation and treatment-response studies. Similar to murine 3D-LTCs, 3D-LTCs from patients with COPD maintained viability as analysed by metabolic assessment (online supplementary fig. S8a) and live/dead staining (online supplementary fig. S8b), and preserved normal or pathophysiological structural changes during culture as assessed by $3 \mathrm{D}$ confocal tissue imaging (fig. $5 \mathrm{a}$ and $\mathrm{b}$ and online supplementary fig. S9). We analysed MMP12 as a disease-specific pathological alteration and found that MMP12 gene expression was significantly increased in 3D-LTC from COPDs patients compared to non-COPD patients (fig. 5c).

Next, we investigated Wnt/ $\beta$-catenin activation in patient-derived 3D-LTCs. Both $\mathrm{Wnt} / \beta$-catenin agonists $\mathrm{LiCl}$ and $\mathrm{CT}$ led to a strong expression of the Wnt/ $\beta$-catenin target genes AXIN2 and NKD1, as well as significantly increased active $\beta$-catenin protein expression (fig. 6), further underpinning that patient-derived $3 \mathrm{D}$-LTCs reflect relevant lung alterations and that $\mathrm{Wnt} / \beta$-catenin signal activation represents a functional target for potential clinical applications.

Initiation of alveolar epithelial cell repair by therapeutic Wnt/B-catenin activation in patient-derived 3D-LTCs leads to alveolar epithelial cell activation

The capability to successfully activate $W n t / \beta$-catenin signalling in patient-derived 3D-LTCs urged us to further explore the cellular effects of $\mathrm{Wnt} / \beta$-catenin activation in human lung tissue. Wnt/ $\beta$-catenin activation by $\mathrm{LiCl}$ 
a)
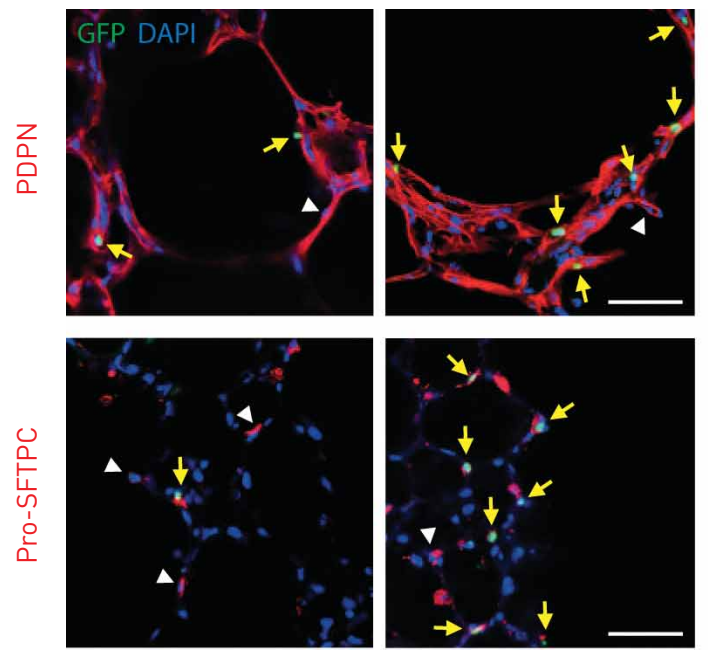
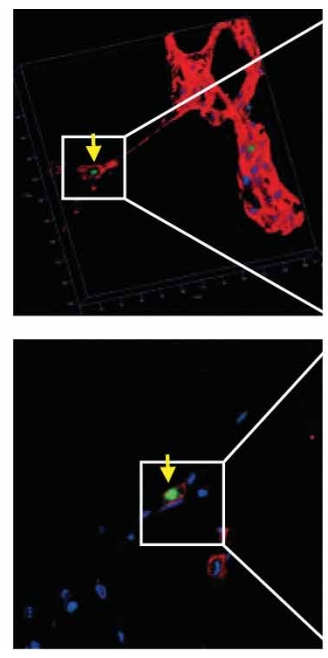
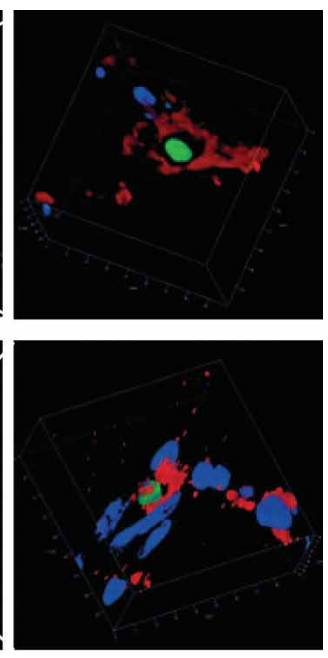

b)

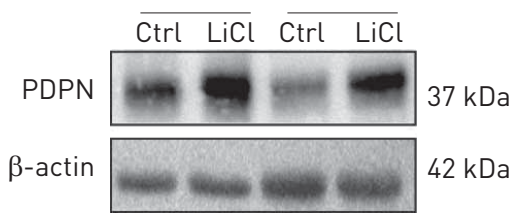

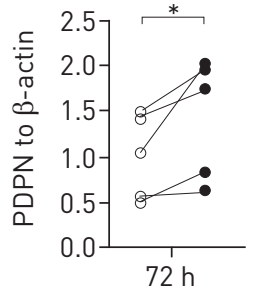

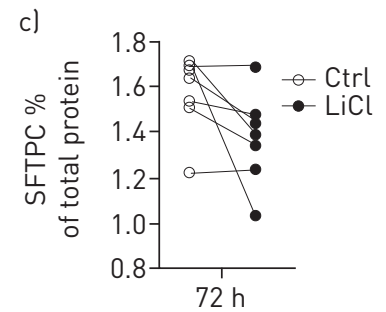

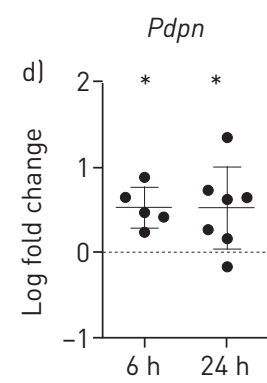
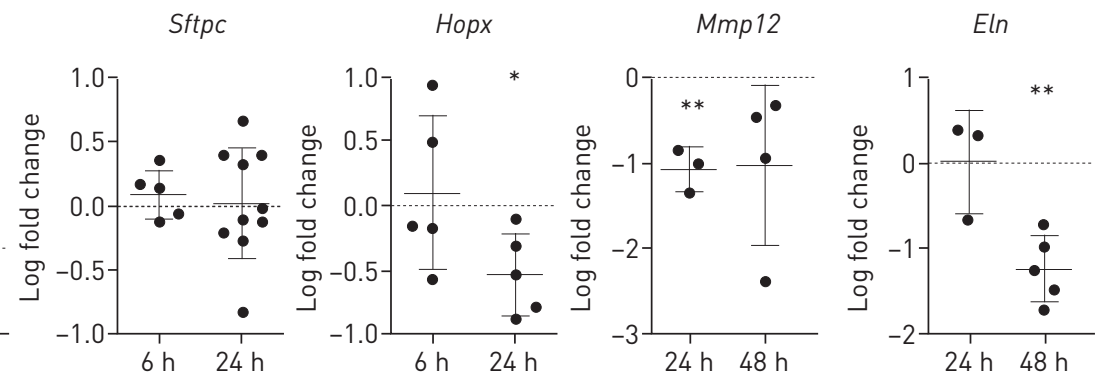

FIGURE 4 Wnt/ $\beta$-catenin activation induces lung epithelial cell markers in three-dimensional (3D) ex vivo tissue cultures (LTCs) from emphysematous mice. a) Representative images of 3D-LTCs from emphysematous TCF/ LEF-EGFP Wnt-reporter animals after $72 \mathrm{~h}$ of treatment with $0.1 \%$ fetal calf serum control medium (Ctrl) or $10 \mathrm{mM}$ lithium chloride (LiCl). Arrows: activated cells expressing green fluorescent protein (GFP); arrowheads: nonactivated podoplanin (PDPN, upper row) and pro-surfactant protein C (pro-SFTPC, lower row)-expressing cells; scale bar $50 \mu \mathrm{m}$. High-power magnification of pro-SFTPC or PDPN, respectively, and GFP-expressing cells (right). Protein expression of alveolar epithelial cell marker PDPN and SFTPC in 3D-LTCs from emphysematous C57Bl/6 mice upon LiCl treatment detected by b) Western blotting and c) ELISA, respectively. d) Gene expression of 3D-LTCs from emphysematous C57BI/6 animals at different time points of treatment with $10 \mathrm{mM} \mathrm{LiCl} \mathrm{compared} \mathrm{to} \mathrm{control:} \mathrm{Pdpn,} \mathrm{Sftpc,} \mathrm{Hopx,} \mathrm{Mmp12} \mathrm{and} \mathrm{Eln.} \mathrm{n=3-10.} \mathrm{*:} \mathrm{p}<0.05$; **: $\mathrm{p}<0.01$.

led to a significant increase in mRNA and protein expression of PDPN, as analysed by qPCR (fig. 7a), Western blotting (fig. 7b) and immunofluorescence staining (fig. 7c). Notably, and in contrast to the murine 3D-LTCs, we also detected an increase in SFTPC mRNA (fig. 7a) and protein (fig. $7 \mathrm{~b}$ and $\mathrm{c}$ ), suggesting that $\mathrm{Wnt} / \beta$-catenin activation stimulates ATII cell function in human $3 \mathrm{D}$-LTCs. These data were further corroborated by decreased levels of HOPX mRNA upon Wnt/ $\beta$-catenin activation by LiCl (fig. 7a). Recently, decreased HOPX expression has been linked to ATII cell maturation $[29,30]$. Together, these observations support the notion that Wnt/ $\beta$-catenin signalling is able to increase ATII and ATI cell marker expression and function in the human diseased lung. Next, we analysed individual tissue response to Wnt/ $\beta$-catenin induced therapy in 3D-LTCs $(n=13)$. Importantly, we found that ATII cell activation as determined by SFTPC significantly correlated with COPD disease stage (as determined by 5 predicted forced expiratory volume in $1 \mathrm{~s}$ (FEV1)) (fig. 7d, SFTPC $\mathrm{p}=0.0079$ ). Similarly, Wnt/ $\beta$-catenin-dependent AXIN2 expression correlated with $\mathrm{FEV} 1 \%$ pred; however, statistical significance was not achieved in this explorative cohort (fig. $7 \mathrm{~d}$ AXIN2 $\mathrm{p}=0.1167$ ). These data strongly 

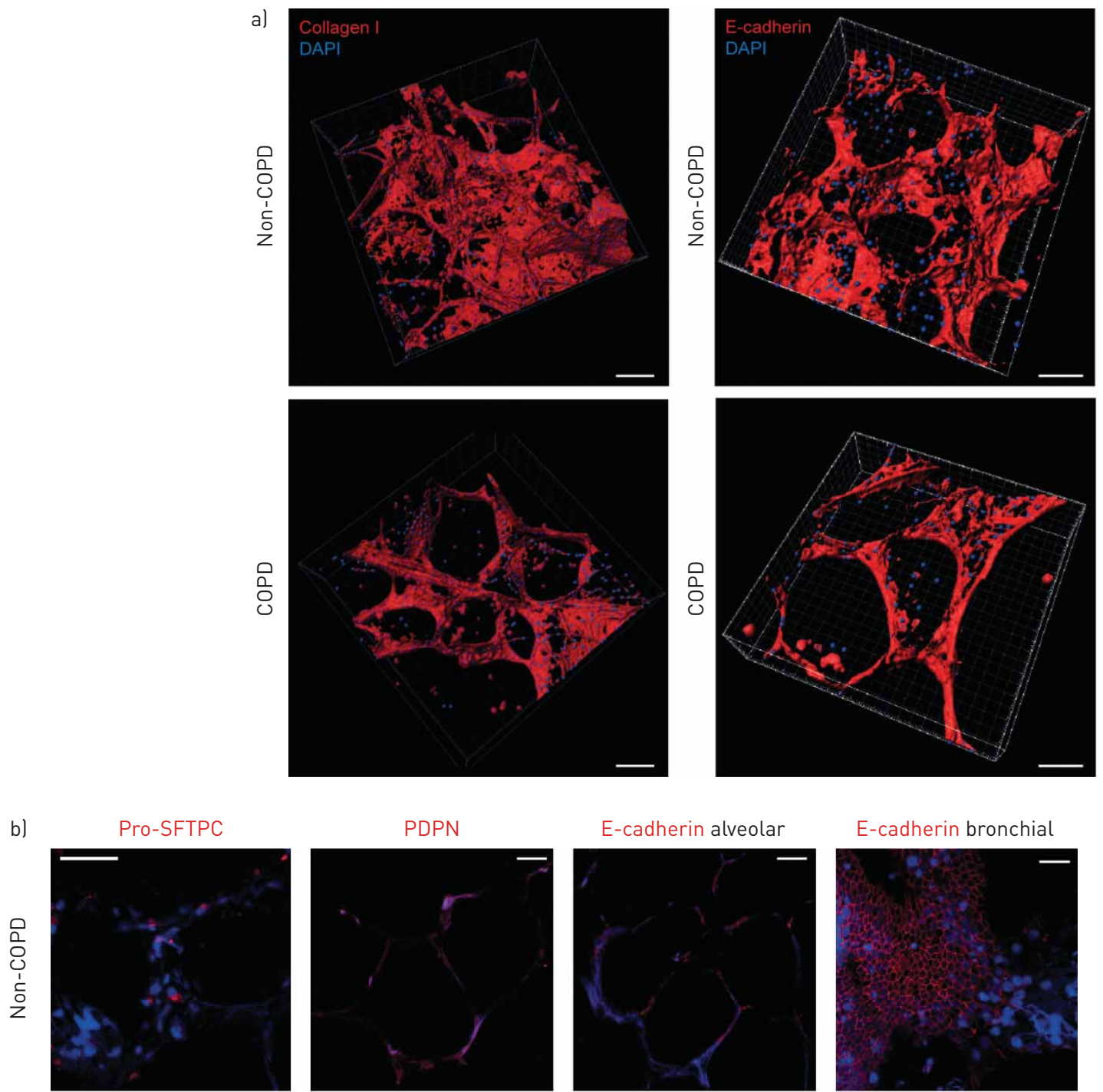

E-cadherin alveolar
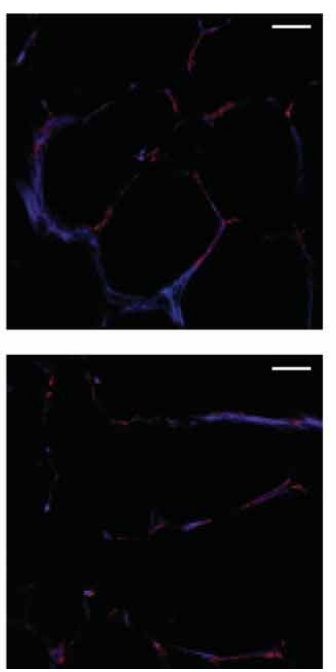

E-cadherin bronchial
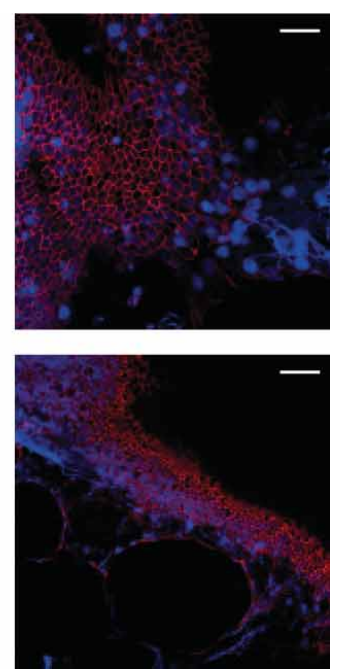
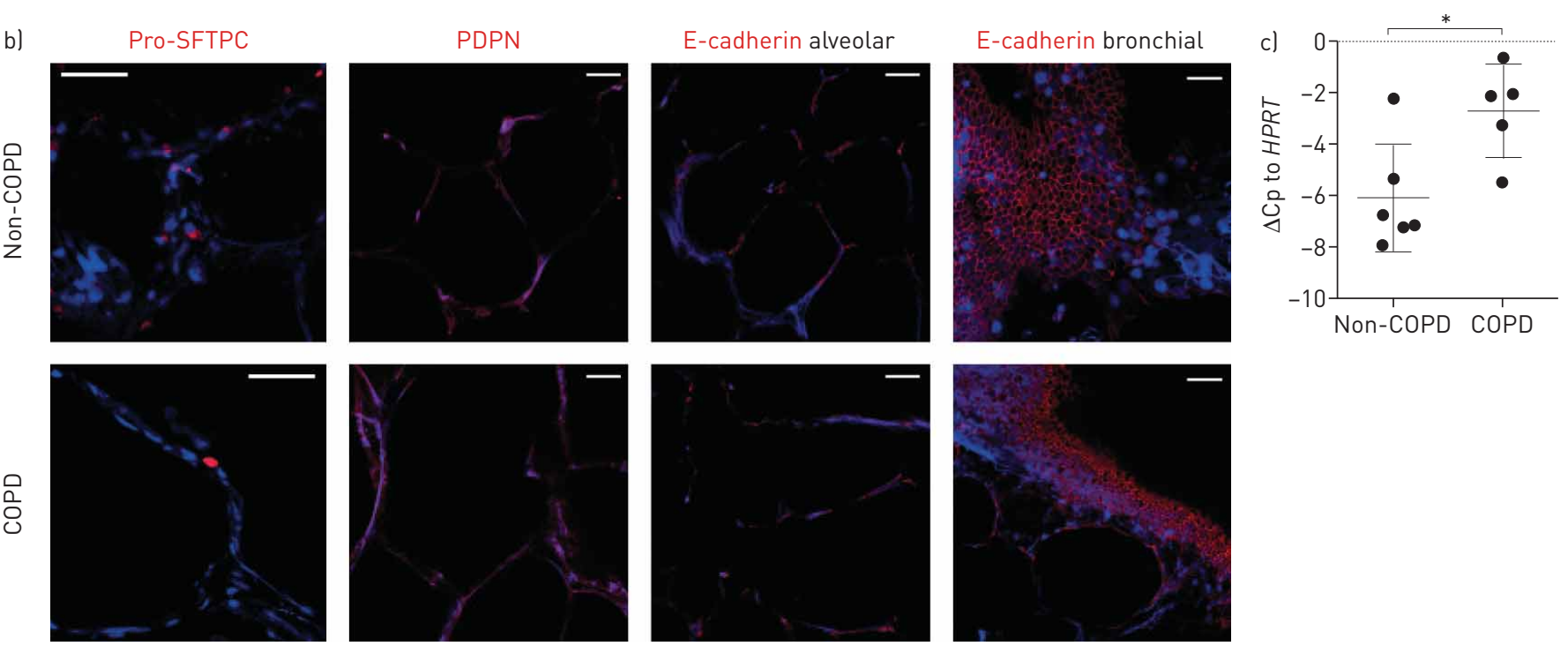

FIGURE 5 Patient-derived three-dimensional (3D) ex vivo tissue cultures (LTCs) remain viable with structural integrity. a) 3D reconstruction of immunofluorescence-labelled 3D-LTCs from non-chronic obstructive pulmonary disease (COPD) and COPD patients. Left: collagen I and right: E-cadherin staining; nuclei were stained with 4'-6-diamidino-2-phenylindole (DAPI); scale bars=100 $\mu$ m. b) Immunofluorescence staining of 3D-LTCs from non-COPD and COPD patients cultivated for $24 \mathrm{~h}$. Pro-surfactant protein C (pro-SFTPC), podoplanin (PDPN) and E-cadherin staining; nuclei were stained blue using DAPI; scale bars $=50 \mu \mathrm{m}$. c) Matrix metalloproteinase 12 gene expression of 3D-LTCs of human non-COPD and COPD patients directly after lung slice generation. $n=5-6 .{ }^{*}: p<0.05$.

suggest that patient-derived 3D-LTCs are valuable to evaluate pharmacological treatment responses in individualised disease stages; however, this needs to be further explored in larger patient cohorts.

Finally, we analysed the result of Wnt/ $\beta$-catenin activation on MMP12 and elastin. While we did not see a significant regulation of MMP12 gene expression (fig. 8a), we observed a significant reduction in tropoelastin protein expression at $72 \mathrm{~h}$ upon $\mathrm{LiCl}$ treatment in human 3D-LTCs (fig. 8b), thus perhaps reflecting a reduced amount of elastin fragments [31]. This was further corroborated by elastin localisation using Hart's stain showing an improved linear deposition of elastin in alveolar walls in human 3D-LTCs upon Wnt/ 
a)
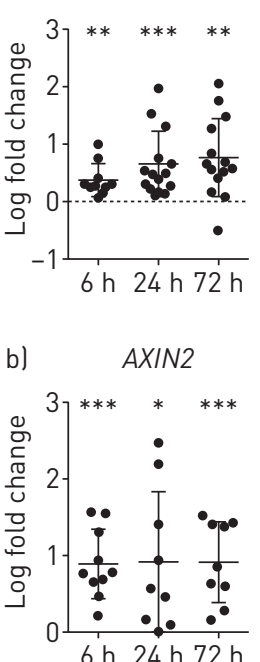

NKD1
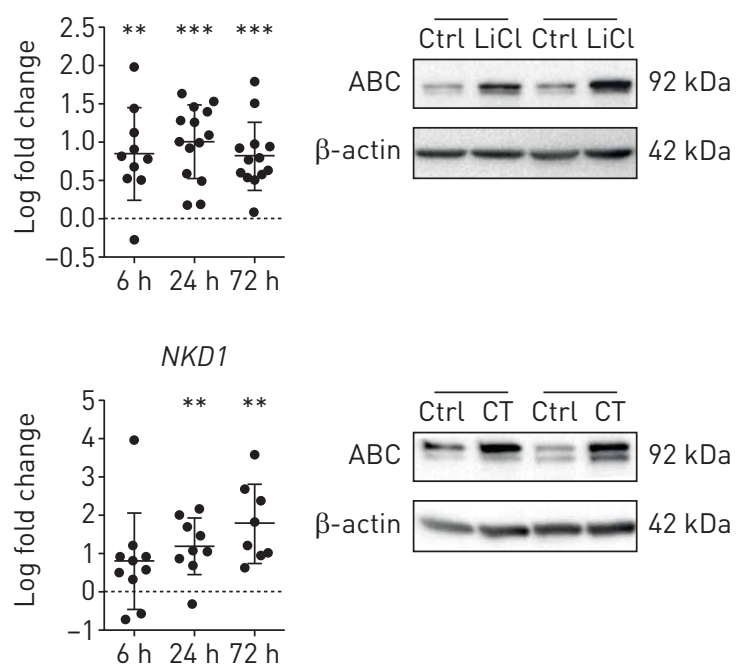
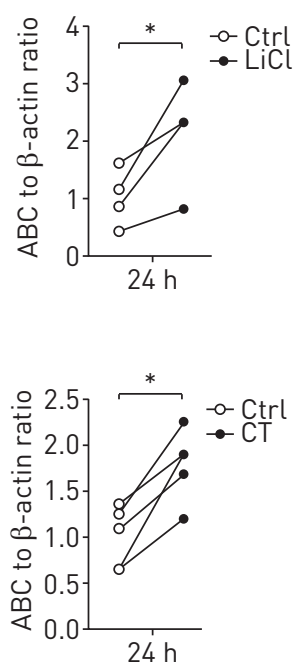

FIGURE $6 \mathrm{Wnt} / \beta$-catenin signalling is inducible in patient-derived three-dimensional (3D) ex vivo tissue cultures (LTCS). Gene expression analysis of Wnt target genes AXIN2 and NKD1 by quantitative PCR and representative Western blotting and densitometry of active $\beta$-catenin $(A B C)$ protein expression in patient-derived 3D-LTCs at the indicated time points after treatment with a) $10 \mathrm{mM}$ lithium chloride (LiCl) or b) $2 \mu \mathrm{M}$ CHIR 99021 (CT). $n=4-14$. Data are presented as mean \pm SD. ${ }^{*}: p<0.05 ;{ }^{* *}: p<0.01 ; * * *: p<0.001$.

$\beta$-catenin activation by $\mathrm{LiCl}$. These findings emphasise that $\mathrm{Wnt} / \beta$-catenin activation also interferes with common pathological processes observed in emphysema patients, and that 3D-LTCs are suitable tools to investigate the effect of potential novel therapies on clinically relevant measures for COPD patients.

\section{Discussion}

COPD is a devastating disease with progressive destruction of functional lung tissue, and only limited symptomatic treatment options are available. Lung transplantation remains the sole therapeutic intervention with a known survival benefit for patients with COPD; however, this is limited mainly by shortage of donor organs. The improvement of donor lung suitability by ex vivo lung perfusion and gene therapy represents a major research area. Nevertheless, novel therapies that target endogenous lung repair processes are urgently needed.

However, reliable and robust tools that allow for proper validation and prediction of potential novel therapies remain sparse. We generated 3D-LTCs from human diseased tissue that can be applied to visualise altered complex lung architecture of diseased lung tissue in 3D with spatiotemporal resolution. We provide proof-of-concept for pathological assessment, drug validation and mechanistic studies in patient-derived 3D-LTCs, which we believe will open novel avenues for successful clinical translation and precision medicine.

While previous reports have used precision-cut lung slices from rodent models and human tissue predominantly for short-term toxicological and pharmacological analysis [32-36], we report the application of 3D-LTCs of patient-derived lungs to study diseased lung tissue and potential lung repair in high spatiotemporal resolution. This represents a major advance in closing the preclinical gap in drug and therapy development, since it allows the evaluation of cell-matrix interaction as well as cellular function in human lung tissue. 3D-LTCs are advantageous because of their versatile application to human patient material. They precisely resemble the matrix and cellular composition of the disease to be studied, in this case COPD, but this can be translated to other lung diseases. We recently demonstrated protease-controlled drug release in human 3D-LTCs from lung cancer patients using mesoporous silica nanoparticles [37]. Despite being incredibly valuable for testing drugs in an intact biological system, in vivo analyses in animal models can only recapitulate our latest understanding of a disease. Therefore, findings from animal studies may not completely resemble the conditions underlying the human disease, showing the importance of studying patient-derived tissue. Here, we demonstrate that major pathological changes are preserved in 3D-LTCs when comparing murine tissue in vivo and ex vivo, which further strengthens the suitability of human 3D-LTCs ex vivo.

We applied 3D-LTCs to show for the first time that Wnt/ $\beta$-catenin signal activation by two different compounds successfully led to the induction of lung repair processes in murine and human 3D-LTCs. To this end, we analysed alveolar epithelial cell function, which has previously been suggested to be affected by 
a)

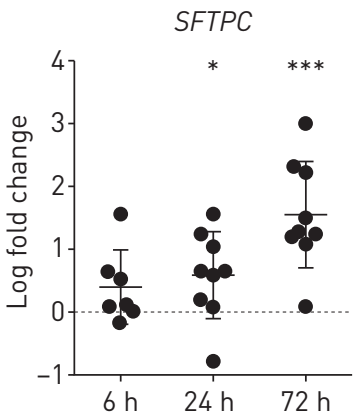

b) i)

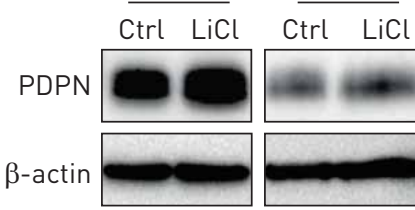

$37 \mathrm{kDa}$

$42 \mathrm{kDa}$

c)
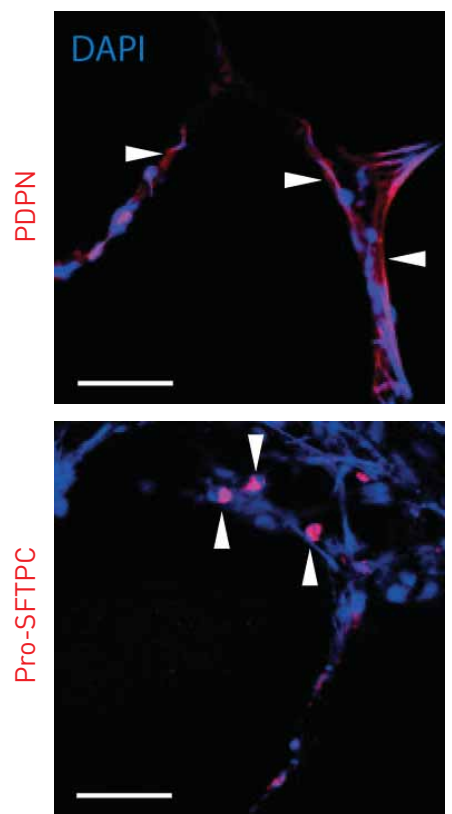

d)

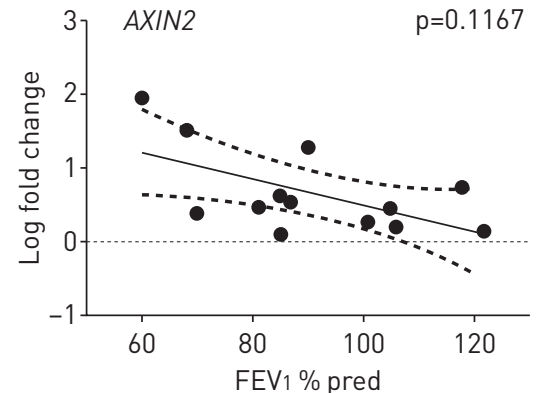

HOPX
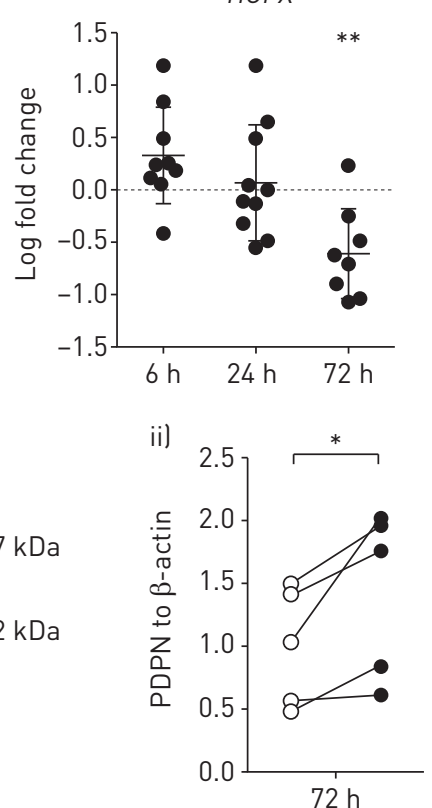

$\mathrm{LiCl}$
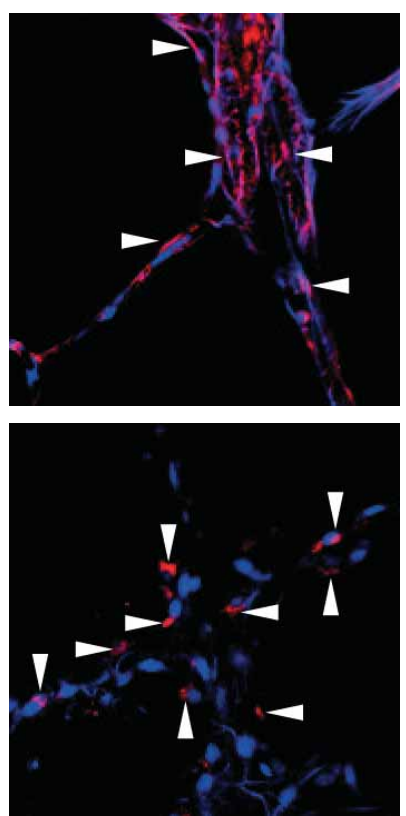
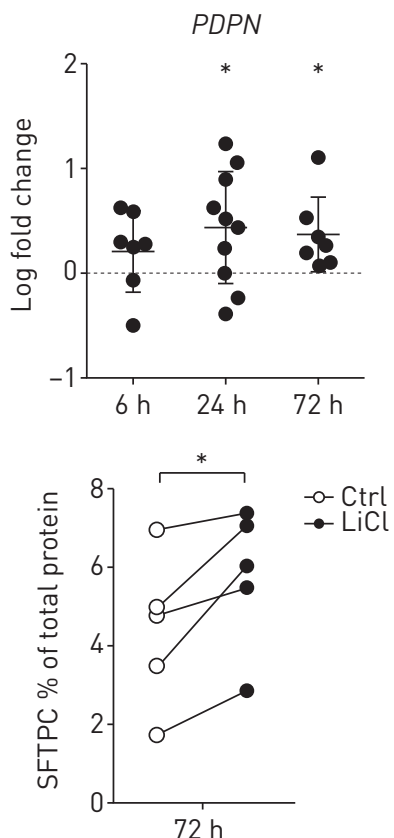

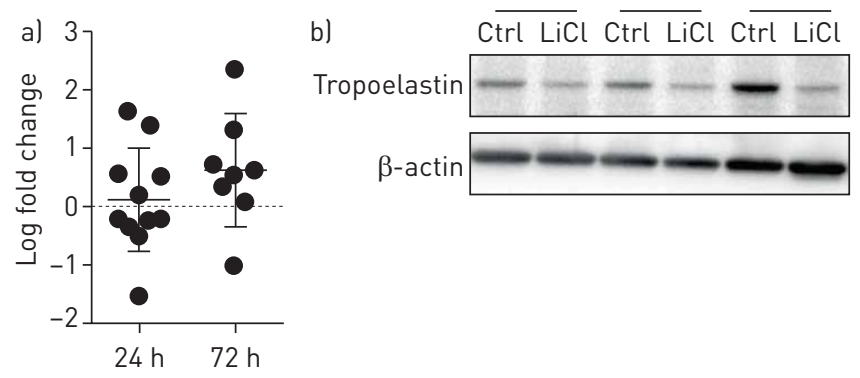

$68 \mathrm{kDa}$
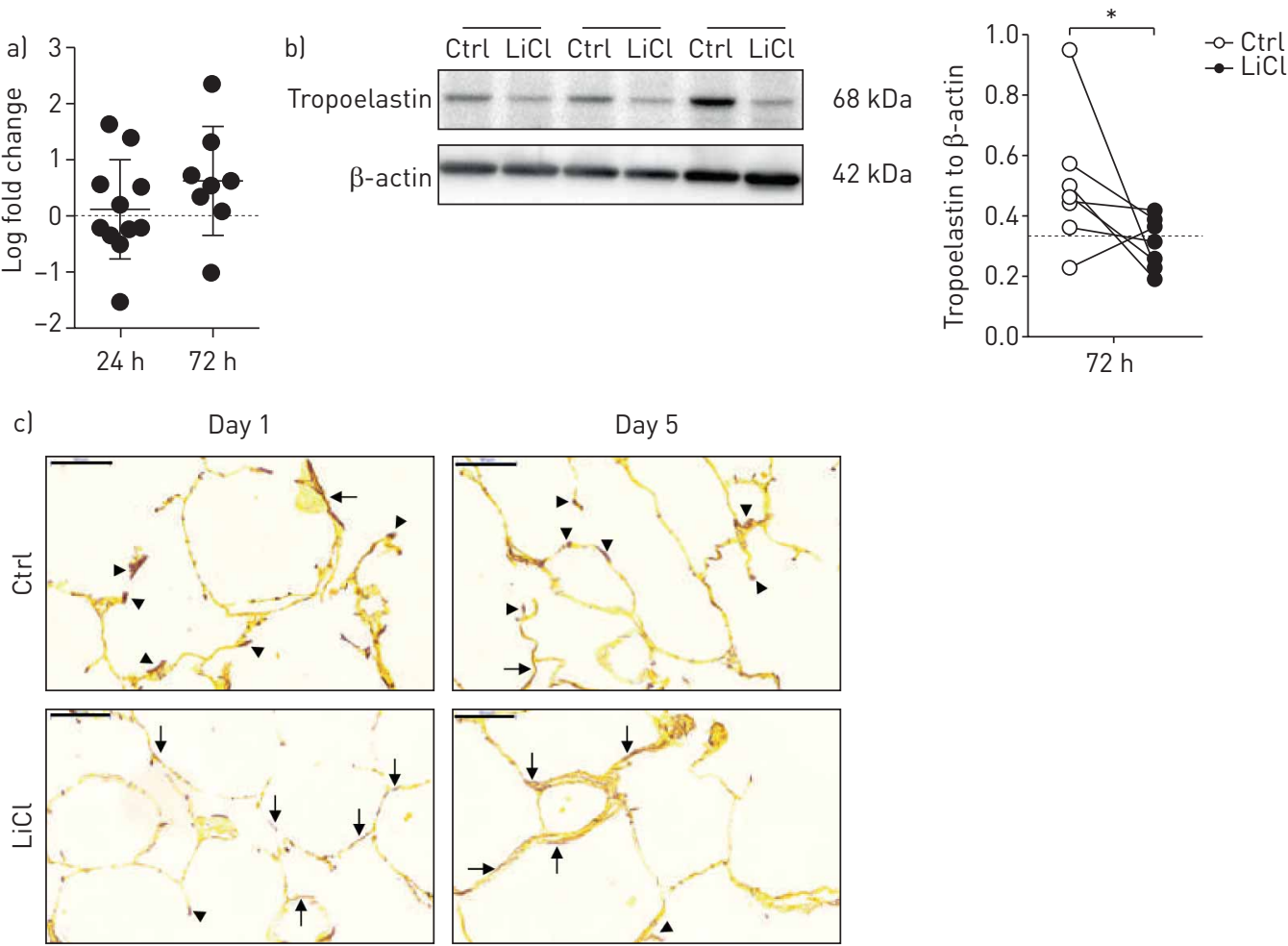

FIGURE 8 Wnt/ $\beta$-catenin activation alters elastin remodelling in patient-derived three-dimensional (3D) ex vivo tissue cultures (LTCs). a) MMP12 gene expression in 3D-LTCs from non-chronic obstructive pulmonary disease (COPD) and COPD patients after treatment with $10 \mathrm{mM} \mathrm{LiCl}$ at different time points; $\mathrm{n}=8-11$. b) Tropoelastin protein expression in patient-derived 3D-LTCs after treatment with $10 \mathrm{mM}$ LiCl for $72 \mathrm{~h} ; \mathrm{n}=7$; $*$ : p $<0.05$. c) Elastin staining of patient-derived 3D-LTCs at indicated time points after LiCl treatment. Arrows show linear deposition of elastin in alveolar walls, while arrowheads show dense, discontinuous deposition; scale bars $=100 \mu \mathrm{m}$.

Wnt/B-catenin activation, as well as well-known pathological measures, such as MMP12 elevation and altered elastin turnover and deposition. Wnt signalling was reduced in 3D-LTCs, similar to that shown in patients with COPD and in vivo animal models of emphysema $[14,15]$. In particular, Wnt/ $\beta$-catenin activation led to differential expression of ATI and ATII cell markers. We found an increase in SFTPC transcript and protein expression upon $\mathrm{Wnt} / \beta$-catenin activation in the patient-derived 3D-LTCs, suggesting that $\mathrm{Wnt} / \beta$-catenin signalling activates ATII cell function or even leads to an increase of the ATII cell population. This increased expression of SFTPC was not detected in 3D-LTCs from emphysematous mice, which might be due to differences in cell/protein turnover and dynamics; however, it further highlights the relevance of using patient-derived tissue for evaluation and validation of promising novel targets and drugs.

Notably, the increase in SFTPC upon Wnt/ß-catenin activation was accompanied by a loss in HOPX expression. It has been recently shown that HOPX is expressed by ATI cells and lost during ATII cells maturation [29]. Reduced HOPX expression, as we have observed upon Wnt/ $\beta$-catenin signal activation, would thus be indicative of an increase in mature and SFTPC producing ATII cells in human lung tissue upon Wnt/ $\beta$-catenin signal activation, rather than a decrease in ATI cells. This notion is supported by an increase in other ATI cell markers upon Wnt/ $\beta$-catenin signal activation, such as podoplanin. These data suggest that Wnt/ $\beta$-catenin activation would stimulate both, ATII and ATI cells in human diseased lung tissue. In line with this, recent evidence by Treutlein and colleagues suggests the existence of bipotent alveolar progenitor cells during murine lung development, giving rise to ATI and ATII cells and expressing markers of both lineages [29]. Potentially, the number of these bipotent alveolar progenitor cells varies between mice and humans and may be one of the reasons for the differences seen in SFTPC expression due to Wnt/ $\beta$-catenin activation. Similarly, we found co-expression of ATII and ATI cell marker as well as single ATI cells expressing podoplanin upon Wnt/ $\beta$-catenin activation. Taken together, these data from mouse and human tissue support the notion that ATI cells might originate not only from ATII cells, but also from other - in human lung tissue, yet to be further defined - alveolar progenitor cells [8, 9, 11, 38]. To proof this unequivocally, future studies are needed that conduct fate-mapping and ex vivo tracking in transgene animals, however, these analyses are limited in human lung tissue with current techniques. 
We found MMP12 to be upregulated in murine and human emphysemateous 3D-LTC, which is in accordance to previous findings of increased MMP12 in COPD patients [26]. Notably, we observed significantly reduced Mmp12 gene expression in the 3D-LTC from emphysematous animals after $24 \mathrm{~h}$ of $\mathrm{LiCl}$ treatment, which went along with reversed Eln transcript level in murine 3D-LTC. Macrophages are one of the main sources for Mmp12 [39]. We further showed reduced macrophage track speed and length in response to $\mathrm{LiCl}$ treatment in emphysematous 3D-LTC suggesting, that Mmp12 reduction might be due to the selective inhibition of macrophage activity. This selective cell action might be involved in the attenuation of emphysema by $\mathrm{LiCl}$ in vivo reported by KNEIDINGER et al. [14], however, the involvement of

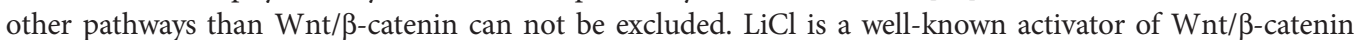
signalling, but can also affect other signalling pathways besides Wnt/ $\beta$-catenin [28]. Here, we confirmed Wnt/ $\beta$-catenin activation and altered Mmp12 and Eln expression in emphysemateous 3D-LTC using the GSK-3 $\beta$ inhibitor CT, which has been described to be a more potent and specific Wnt/ $\beta$-catenin activator [21]. The detailed signalling mechanisms as well as potential contributions of other cell types, such as neutrophils, B-cells, T-cells, and endothelial cells, needs to be examined in future studies.

In human 3D-LTC, we did not observe significant changes of MMP12 transcript in patient-derived 3D-LTC. This might be due to the limited number of samples, which we were able to analyse in this proof-of-concept study. However, we observed decreased tropoelastin expression in 3D-LTC lysates, which might refer to a reduction of chemotactic activity and elastin fragments after LiCl treatment. Mature elastin consists of various tropoelastin monomers irreversibly cross-linked via lysyl oxidases. Proteolysis of elastin by serine proteases such as neutrophil elastase or MMP12 results in elastin fragments of multiple size and composition. It has been shown previously, that MMP- $12^{-/-}$mice are protected from the development of cigarette smoke (CS)-induced emphysema via a reduction in elastin fragment generation and thus, less monocyte chemotactic activity in BALF of these mice compared to control [25]. Recent studies have further highlighted reduced lysyl oxidase expression and fibulin-5 activity contributing to impaired elastic fibre assembly and repair in COPD patients [40, 41]. In this context, a reduction of tropoelastin might reduce the number of misassembled elastin fibres (and therefore reduce protein overload) and the potential source of monocyte chemotactic activity. That $\mathrm{LiCl}$ treatment positively interferes with major pathological processes in COPD is further supported by Hart's staining of human 3D-LTC, which demonstrated an increase in linear elastin deposition in the alveolus.

It should be noted that in order to fully determine the capacity of lung repair or even re-growth also in human tissue, patient-derived 3D-LTCs most likely need to be analysed for longer time points and advanced culture systems might be considered. Our ex vivo system currently lacks several relevant in vivo inputs such as perfusion through blood vessels, the presence of an air-liquid interface (i.e. cellular-level control of gas levels), and mechanical stretch. Another potential difference in our system from the in vivo environment stems from the lack of a renewable supply of immune cells. While we still observe immune cells in our system, we have yet to fully characterise the activity of these cells (e.g. polarisation). Furthermore, other experimental parameters such as the media composition and use of specific growth factors could be critical in studying specific cell populations or tissue level responses. All of these parameters have been previously shown to have profound effects on cellular and tissue level behaviour, including repair and regeneration [42]. Optimisation and further understanding the role of these parameters will be important in maximising usage of 3D-LTC.

For our murine 3D-LTC studies, we used the well-characterised model of elastase-induced emphysema, which exhibits airspace enlargement and has been shown to exhibit reduced Wnt/ $\beta$-catenin signalling in vivo [14]. This model does not reflect pathological features, including chronic bronchitis, which is present in patients with COPD [2]. Here, we used peripheral tissue for our patient-derived 3D-LTC and largely concentrate on the role of alveolar epithelial cells however, it has been reported that next to alveolar epithelial cell injury, endothelial cell impairment and apoptosis might occur in emphysema [6, 7]. While we did not observe a large number of $\mathrm{Wnt} / \beta$-catenin activated endothelial cells in emphysematous 3D-LTC in the current study, it will be important for future studies to further explore the role of Wnt/ $\beta$-catenin signalling on other main mechanisms contributing to alveolar tissue destruction.

Moreover, studies with murine 3D-LTC exposed to cigarette smoke or derived from animals that have been subjected to cigarette smoke in vivo will be useful to further determine and validate promising targets and drugs for COPD. One advantage of 3D-LTC in this context is the possibility to visualise and track cells in their natural environment by $4 \mathrm{D}$ confocal live tissue imaging, which we have applied in this study to visualise $\mathrm{Wnt} / \beta$-catenin activation in lung cells over time (Video 2 ), as well as to track macrophage movements upon Wnt/ $\beta$-catenin activation (Video 3 and 4). Moreover, the usage of several adjacent slices with similar cellular composition and lung architecture is advantageous and allows a more accurate assessment of therapeutic effects, since slices from the same sample can be used for treatment and control. 
Thereby, 3D-LTC further benefit from the possibility to analyse various readouts from a small amount of tissue and, when using animal models, thus reduce overall animal experimentation.

In the present study, we found a significant correlation of increased SFTPC gene expression following $\mathrm{LiCl}$ treatment with disease stage of the patient, which is clinically largely determined by lung function. Wnt/ $\beta$-catenin signal activation analysed by increased AXIN2 expression with $\mathrm{LiCl}$ treatment, followed the same trend without reaching statistical significance (fig. 7d). Notably, we observed a stronger increase in ATII cell marker expression in patients with more severe COPD, indicating that these patients do not only have some lung epithelial cell repair capacity, but could benefit in particular. Thus, 3D-LTC represent a valuable tool for personalised medicine tailoring treatments to the individual requirements of the patient, and thereby advancing drug testing before clinical studies are conducted. Moreover, several compounds can be tested in parallel on serial sections, which could lead to more robust and reliable outcomes. While this study aimed to provide proof-of-concept of the suitability of 3D-LTC, the number of patient samples was limited. Future studies with larger cohorts particularly of non-COPD as well as COPD patients are needed to further analyse disease heterogeneity and validate the disease specificity of our findings.

One major advantage of patient-derived 3D-LTC used in this study, is the analysis of the complex 3D architecture and endogenous cellular function and cell-type composition of patient-derived tissue. Other applications of the basic lung slice technology include the study of decellularised slices, which can be used to investigate exogenously seeded cell behaviour, such as the role of extracellular matrix and mechanical properties for cell fate decisions $[43,44]$ and as a tool for both studying and directing human pluripotent stem cell differentiation into lung epithelial cells [45]. Similarly, HuH et al. [46] developed a "lung-on-a-chip" micro-device to model the alveolar-capillary interface. This approach has been recently also used to create a system to study in vivo-like alveolar microinjuries and wound repair [47]. These models, which are mainly based on the use of cell lines, can and, in part, have been successfully applied for robust high-throughput drug discovery. However, to further analyse complex physiological responses and drug efficiency patient-derived native lung tissue is needed. Here, we focussed on the endogenous repair capacity of the human diseased lung and revealed insight into cellular function and the capability of the remaining lung to respond to novel treatment options, such as Wnt- $\beta$-catenin induced lung repair. We believe that this is of particular value to determine and predict the suitability of novel targets and drugs to enter clinical trials.

In summary, these findings underline that 3D-LTC not only represent a valuable tool for preclinical drug evaluation and treatment responses, but are further suitable to investigate essential disease related mechanism in $3 \mathrm{D}$ with spatio-temporal resolution. Therefore, we regard the model of 3D-LTC as an important bridging technology for drug identification, evaluation, treatment prediction, and personalised medicine.

\section{Acknowledgments}

We thank all members of the Königshoff Lab for stimulating discussions and critical reading of the manuscript. We are especially indebted to Nadine Adam (Comprehensive Pneumology Center, Munich) as well as Anja Stowasser (Asklepios Clinic, Gauting), Diana Steinhart (Asklepios Clinic, Gauting), Maria Magdalena Stein (Comprehensive Pneumology Center, Munich), Maria Neuner (Comprehensive Pneumology Center, Munich), and Andrea Naujok (Comprehensive Pneumology Center, Munich) for excellent technical assistance. All the members of the small animal facility of the institute are thanked for their constant support and help. We also like to thank Tobias Stoeger (Comprehensive Pneumology Center, Munich) for providing the MacGreen animals.

\section{References}

1 Stockley RA, Mannino D, Barnes PJ. Burden and pathogenesis of chronic obstructive pulmonary disease. Proc Am Thorac Soc 2009; 6: 524-526.

2 Churg A, Wright JL. Animal models of cigarette smoke-induced chronic obstructive lung disease. Contrib Microbiol 2007; 14: 113-125.

3 Rabe KF, Hurd S, Anzueto A, et al. Global strategy for the diagnosis, management, and prevention of chronic obstructive pulmonary disease: GOLD executive summary. Am J Respir Crit Care Med 2007; 176: 532-555.

4 Chung KF, Adcock IM. Multifaceted mechanisms in COPD: inflammation, immunity, and tissue repair and destruction. Eur Respir J 2008; 31: 1334-1356.

5 Hogg JC, Timens W. The pathology of chronic obstructive pulmonary disease. Annu Rev Pathol 2009; 4: 435-459. Tuder RM, Petrache I. Pathogenesis of chronic obstructive pulmonary disease. I Clin Invest 2012; 122: 2749-2755. Taraseviciene-Stewart L, Voelkel NF. Molecular pathogenesis of emphysema. J Clin Invest 2008; 118: 394-402. Kotton DN, Morrisey EE. Lung regeneration: mechanisms, applications and emerging stem cell populations. Nat Med 2014; 20: 822-832.

9 Rock J, Königshoff M. Endogenous lung regeneration: potential and limitations. Am J Respir Crit Care Med 2012; 186: 1213-1219.

10 Barkauskas CE, Cronce MJ, Rackley CR, et al. Type 2 alveolar cells are stem cells in adult lung. J Clin Invest 2013; 123: 3025-3036.

11 Hogan BL, Barkauskas CE, Chapman HA, et al. Repair and regeneration of the respiratory system: complexity, plasticity, and mechanisms of lung stem cell function. Cell Stem Cell 2014; 15: 123-138. 

repair. Eur Respir J 2004; 24: 664-673.

Goss AM, Tian Y, Tsukiyama T, et al. Wnt2/2b and $\beta$-catenin signaling are necessary and sufficient to specify lung progenitors in the foregut. Dev Cell 2009; 17: 290-298.

14 Kneidinger N, Yildirim AO, Callegari J, et al. Activation of the WNT/ $\beta$-catenin pathway attenuates experimental emphysema. Am J Respir Crit Care Med 2011; 183: 723-733.

15 Wang R, Ahmed J, Wang G, et al. Down-regulation of the canonical Wnt $\beta$-catenin pathway in the airway epithelium of healthy smokers and smokers with COPD. PLoS One 2011; 6: e14793.

16 Miller WR, Larionov AA. Bridging the gap between translational research and clinical application. J Natl Cancer Inst Monogr 2011; 2011: 134-137.

17 Forbes SJ, Rosenthal N. Preparing the ground for tissue regeneration: from mechanism to therapy. Nat Med 2014; 20: $857-869$.

18 Maretto S, Cordenonsi M, Dupont S, et al. Mapping Wnt/ $\beta$-catenin signaling during mouse development and in colorectal tumors. Proc Natl Acad Sci USA 2003; 100: 3299-3304

19 Ferrer-Vaquer A, Piliszek A, Tian G, et al. A sensitive and bright single-cell resolution live imaging reporter of Wnt/ $\beta$-catenin signaling in the mouse. BMC Dev Biol 2010; 10: 121.

20 Hedgepeth CM, Conrad LJ, Zhang J, et al. Activation of the Wnt signaling pathway: a molecular mechanism for lithium action. Dev Biol 1997; 185: 82-91.

21 Bain J, Plater L, Elliott M, et al. The selectivity of protein kinase inhibitors: a further update. Biochem J 2007; 408: 297-315.

22 Königshoff M, Kramer M, Balsara N, et al. WNT1-inducible signaling protein-1 mediates pulmonary fibrosis in mice and is upregulated in humans with idiopathic pulmonary fibrosis. J Clin Invest 2009; 119: 772-787.

23 Königshoff M, Eickelberg O. WNT signaling in lung disease: a failure or a regeneration signal? Am J Respir Cell Mol Biol 2010; 42: 21-31.

24 Chilosi M, Poletti V, Zamò A, et al. Aberrant Wnt/ $\beta$-catenin pathway activation in idiopathic pulmonary fibrosis. Am J Pathol 2003; 162: 1495-1502.

25 Houghton AM, Quintero PA, Perkins DL, et al. Elastin fragments drive disease progression in a murine model of emphysema. J Clin Invest 2006; 116: 753-759.

26 Demedts IK, Morel-Montero A, Lebecque S, et al. Elevated MMP-12 protein levels in induced sputum from patients with COPD. Thorax 2006; 61: 196-201.

27 Kahn M. Can we safely target the WNT pathway? Nat Rev Drug Discov 2014; 13: 513-532.

28 De Meyer I, Martinet W, Van Hove CE, et al. Inhibition of inositol monophosphatase by lithium chloride induces selective macrophage apoptosis in atherosclerotic plaques. Br J Pharmacol 2011; 162: 1410-1423.

29 Treutlein B, Brownfield DG, Wu AR, et al. Reconstructing lineage hierarchies of the distal lung epithelium using single-cell RNA-seq. Nature 2014; 509: 371-375.

30 Yin Z, Gonzales L, Kolla V, et al. Hop functions downstream of Nkx2.1 and GATA6 to mediate HDAC-dependent negative regulation of pulmonary gene expression. Am J Physiol Lung Cell Mol Physiol 2006; 291: L191-L199.

31 Senior RM, Griffin GL, Mecham RP. Chemotactic responses of fibroblasts to tropoelastin and elastin-derived peptides. J Clin Invest 1982; 70: 614-618.

32 de Jong M, Essers J, van Weerden WM. Imaging preclinical tumour models: improving translational power. Nat Rev Cancer 2014; 14: 481-493.

33 Liberati TA, Randle MR, Toth LA. In vitro lung slices: a powerful approach for assessment of lung pathophysiology. Expert Rev Mol Diagn 2010; 10: 501-508.

34 Parrish AR, Gandolfi AJ, Brendel K. Precision-cut tissue slices: applications in pharmacology and toxicology. Life Sci 1995; 57: 1887-1901.

35 Sewald K, Braun A. Assessment of immunotoxicity using precision-cut tissue slices. Xenobiotica 2013; 43: 84-97.

36 Siminski JT, Kavanagh TJ, Chi E, et al. Long-term maintenance of mature pulmonary parenchyma cultured in serum-free conditions. Am J Physiol 1992; 262: L105-L110.

37 van Rijt SH, Bölükbas DA, Argyo C, et al. Protease-mediated release of chemotherapeutics from mesoporous silica nanoparticles to ex vivo human and mouse lung tumors. ACS Nano 2015; 9: 2377-2389.

38 Fujino N, Kubo H, Suzuki T, et al. Isolation of alveolar epithelial type II progenitor cells from adult human lungs. Lab Invest 2011; 91: 363-378.

39 Hautamaki RD, Kobayashi DK, Senior RM, et al. Requirement for macrophage elastase for cigarette smoke-induced emphysema in mice. Science 1997; 277: 2002-2004.

40 Li W, Zhou J, Chen L, et al. Lysyl oxidase, a critical intra- and extra-cellular target in the lung for cigarette smoke pathogenesis. Int J Environ Res Public Health 2011; 8: 161-184.

41 Brandsma CA, van den Berge M, Postma DS, et al. A large lung gene expression study identifying fibulin-5 as a novel player in tissue repair in COPD. Thorax 2015; 70: 21-32.

42 Wagner DE, Bonvillain RW, Jensen T, et al. Can stem cells be used to generate new lungs? Ex vivo lung bioengineering with decellularized whole lung scaffolds. Respirology 2013; 18: 895-911.

43 Wagner DE, Bonenfant NR, Sokocevic D, et al. Three-dimensional scaffolds of acellular human and porcine lungs for high throughput studies of lung disease and regeneration. Biomaterials 2014; 35: 2664-2679.

44 Booth AJ, Hadley R, Cornett AM, et al. Acellular normal and fibrotic human lung matrices as a culture system for in vitro investigation. Am J Respir Crit Care Med 2012; 186: 866-876.

45 Huang SX, Islam MN, O’Neill J, et al. Efficient generation of lung and airway epithelial cells from human pluripotent stem cells. Nat Biotechnol 2014; 32: 84-91.

46 Huh D, Leslie DC, Matthews BD, et al. A human disease model of drug toxicity-induced pulmonary edema in a lung-on-a-chip microdevice. Sci Transl Med 2012; 4: 159ra147.

47 Felder M, Stucki AO, Stucki JD, et al. The potential of microfluidic lung epithelial wounding: towards in vivo-like alveolar microinjuries. Integr Biol 2014; 6: 1132-1140. 